\title{
QRPA calculations of stellar weak-interaction rates
}

P. Sarriguren

Instituto de Estructura de la Materia

CSIC, Madrid, Spain

IEM $=$ CSIC

Zakopane Conference on Nuclear Physics:

Extremes of Nuclear Landscape.

August 28 - September 4 (2016) 


\section{Weak-decay rates}

\section{Problem :}

$>$ Weak-decay rates determine late stages of stellar evolution.

$>$ Experimental extrapolations or theoretical predictions:

Reproduce exp information on $T_{1 / 2}$ and $B G T$ under terrestrial conditions.

\section{Theoretical approach :}

$>$ Deformed HF+BCS+QRPA formalism with Skyrme forces and residual interactions in both ph and pp channels.

Results : Weak-decay rates at various $(\rho, T)$ in stellar scenarios

$>$ pf-shell nuclei. Main constituents of stellar core in presupernova formations: Sc, Ti, V, Mn, Fe, Co, Ni, Zn isotopes.

BGT measured in laboratory (Charge exchange reactions) and compared with benchmark Shell Model calculations.

$>$ Neutron rich $\mathrm{Zr}$-Mo isotopes: $r$ process.

$>$ Neutron-deficient waiting-point isotopes $(\mathrm{Ni}-\mathrm{S} n)$ at $(\rho, T)$ typical of rpprocess. 


\section{Weak-decay rates}

$$
\lambda=\ln 2\left(T_{1 / 2}\right)^{-1}=\frac{\ln 2}{D} \sum_{\text {if }} P_{i}(T) B_{i f} \Phi_{i f}(\rho, T)
$$

Initial states thermally populated

$$
P_{i}(T)=\frac{2 J_{i}+1}{G} e^{-E_{i} /(k T)}, \quad G=\sum_{i}\left(2 J_{i}+1\right) e^{-E_{i} /(k T)} P_{i=g . s .}(T=0)=1
$$

Nuclear structure

$$
B_{i f}(G T)=\left(\frac{g_{A}}{g_{V}}\right)_{\mathrm{eff}}^{2}\left\langle f\left\|\sum_{k} \sigma^{k} t_{ \pm}^{k}\right\| i\right\rangle^{2}
$$

Phase space factors :

$$
\beta^{+}, E C: \Phi_{i f}=\Phi_{i f}^{E C}+\Phi_{i f}^{\beta^{+}} \quad \beta^{-}: \Phi_{i f}=\Phi_{i f}^{\beta^{-}}
$$

$\lambda(\rho, T)$ are different from laboratory $\left(P_{i}, c E C\right)$ 


\section{Phase space factors}

$\Phi_{i f}^{\beta^{-}}=\int_{1}^{Q_{i f}} \omega \sqrt{\omega^{2}-1}\left(Q_{i f}-\omega\right)^{2} F(Z+1, \omega)\left[1-S_{e}(\omega)\right]\left[1-S_{v}\left(Q_{i f}-\omega\right)\right] d \omega$

$\Phi_{i f}^{\beta^{+}}=\int_{1}^{Q_{i f}} \omega \sqrt{\omega^{2}-1}\left(Q_{i f}-\omega\right)^{2} F(-Z+1, \omega)\left[1-S_{p}(\omega)\right]\left[1-S_{v}\left(Q_{i f}-\omega\right)\right] d \omega$

$\Phi_{i f}^{c E C}=\int_{\omega_{\ell}}^{\infty} \omega \sqrt{\omega^{2}-1}\left(Q_{i f}+\omega\right)^{2} F(Z, \omega) S_{e}(\omega)\left[1-S_{v}\left(Q_{i f}+\omega\right)\right] d \omega$

$\Phi^{o E C}=\frac{\pi}{2}\left[q_{K}^{2} g_{K}^{2} B_{K}+q_{L_{1}}^{2} g_{L_{1}}^{2} B_{L_{1}}+\cdots\right]\left[\begin{array}{ll}q & \text { Neutrino energy } \\ g & \text { Radial components of the e-wf at } r=0 \\ B & \text { Exchange and overlap corrections }\end{array}\right.$

Distribution functions

$S_{p}=S_{v}=0$

$S_{e}$ : Fermi-Dirac distribution

$S_{e}=\frac{1}{\exp \left[\left(\omega-\mu_{e}\right) /(k T)\right]+1}$
$Q_{i f}=\frac{1}{m_{e} c^{2}}\left(M_{p}-M_{d}+E_{i}-E_{f}\right)$

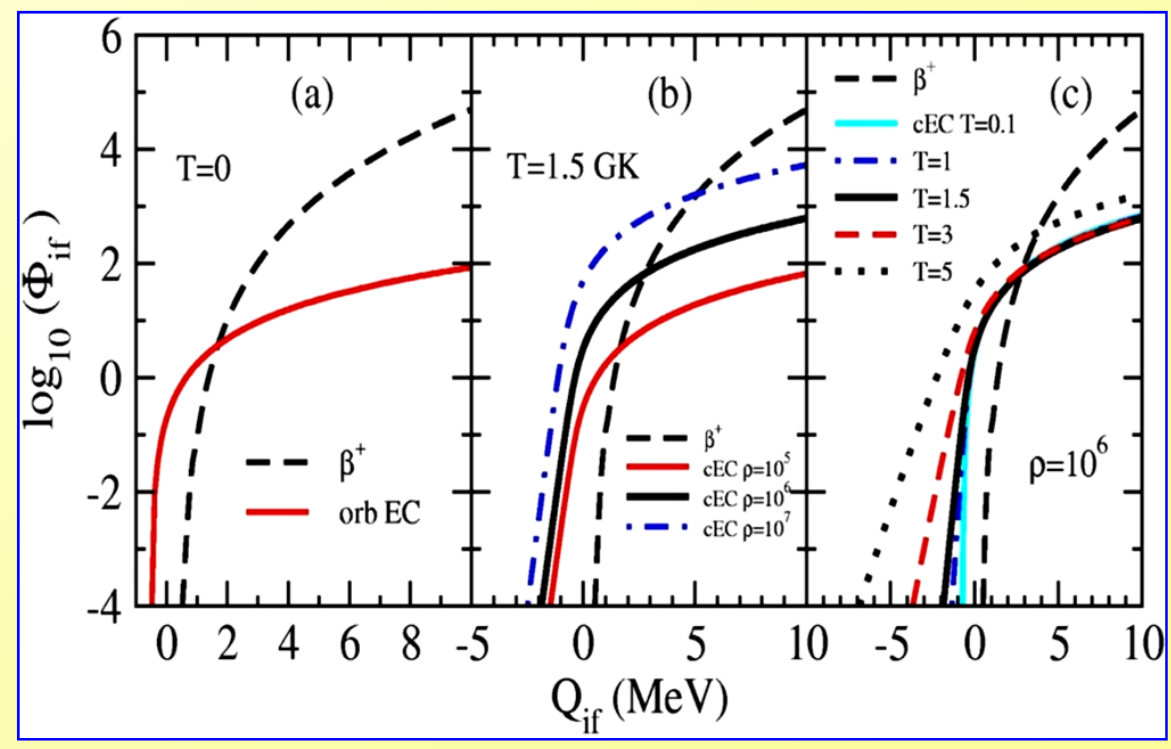




\section{Nuclear Structure}

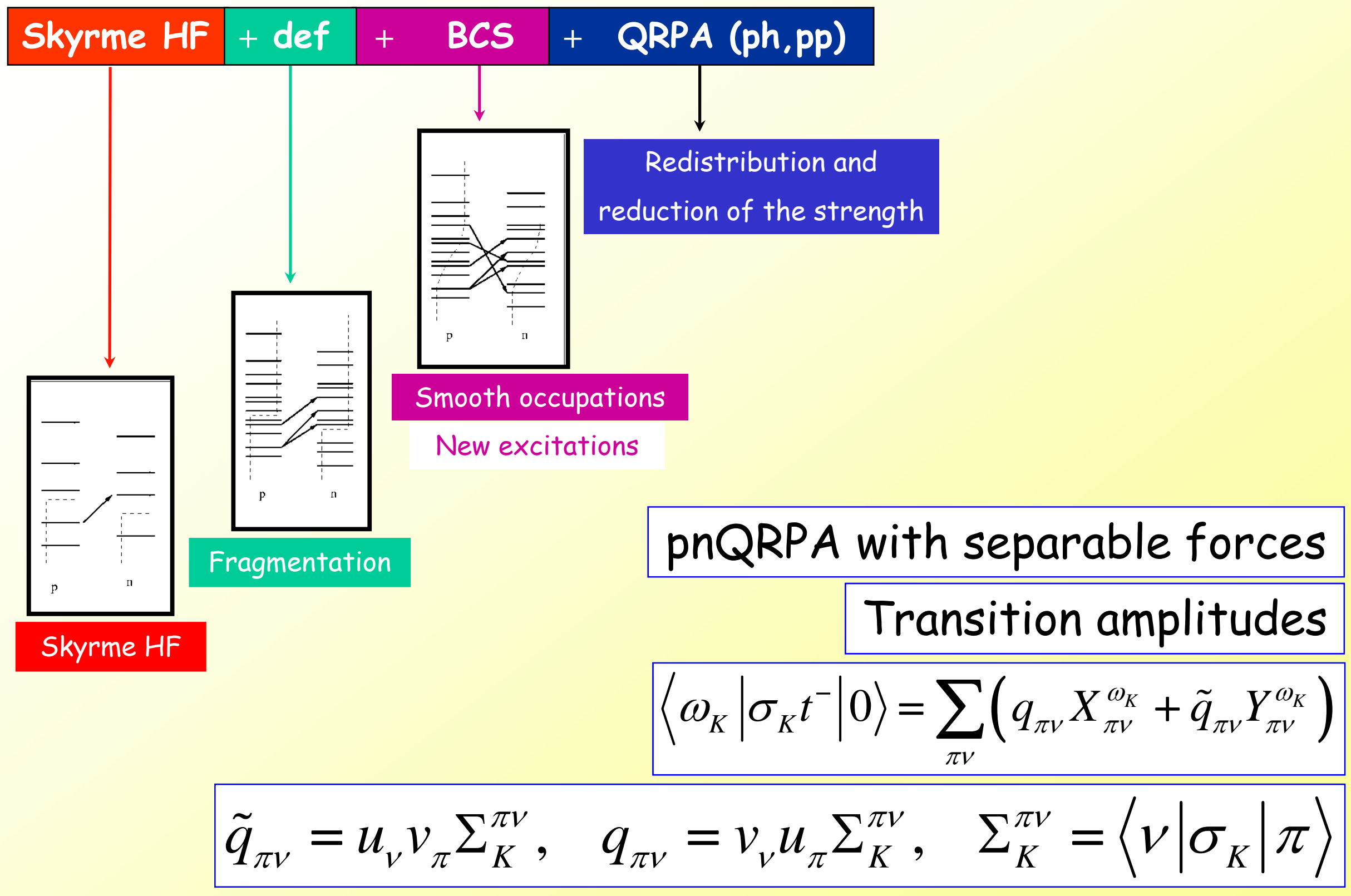




\section{Neutron-rich isotopes: $\mathrm{Ge}-\mathrm{Pd}$}

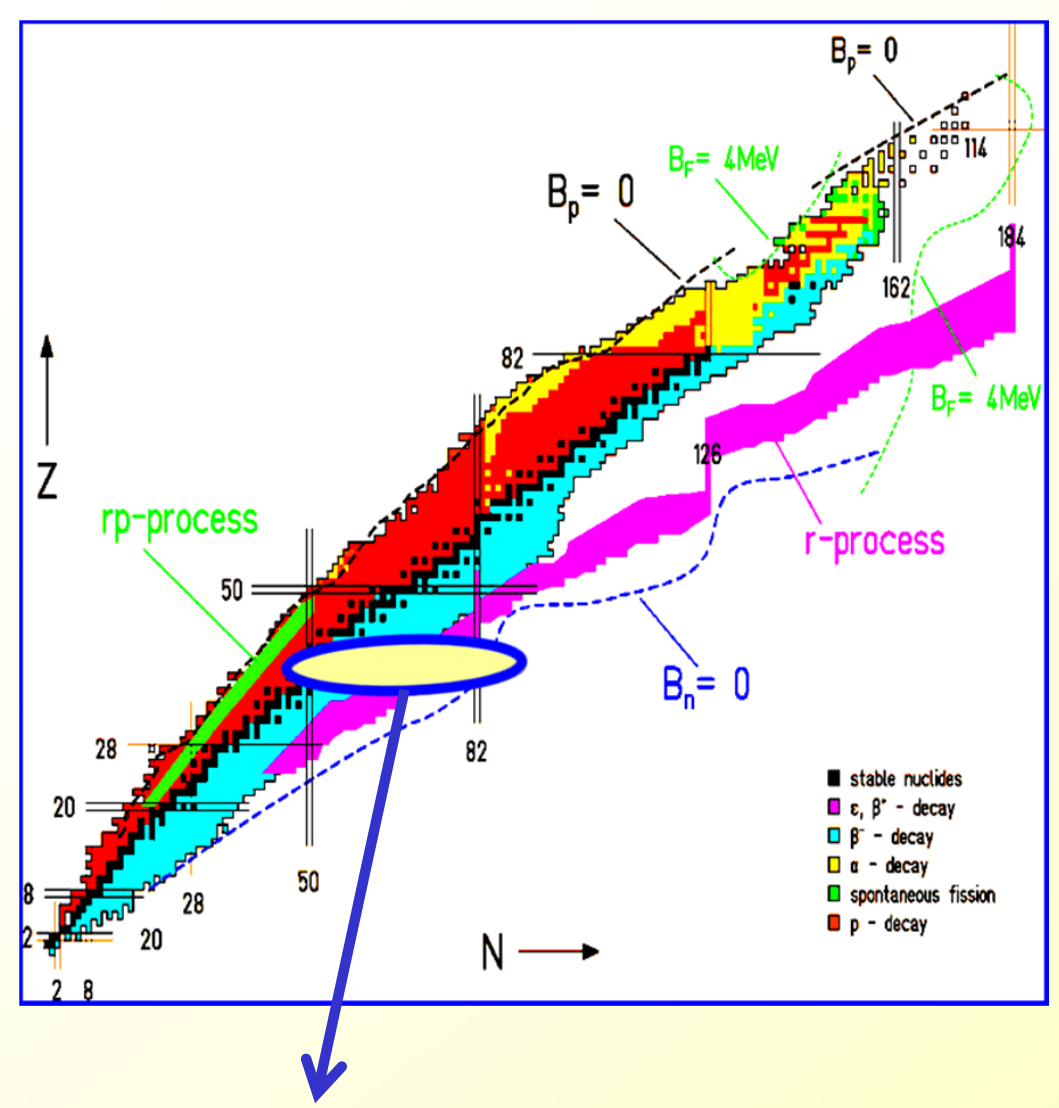

80-94Ge, ${ }^{86-100} \mathrm{Se},{ }^{90-104} \mathrm{Kr},{ }^{94-108} \mathrm{Sr}$,

100-114Zr, 104-118Mo, 110-124Ru, 114-128Pd

r-process: supernovae, $n$-star mergers Nucleosynthesis of heavy elements

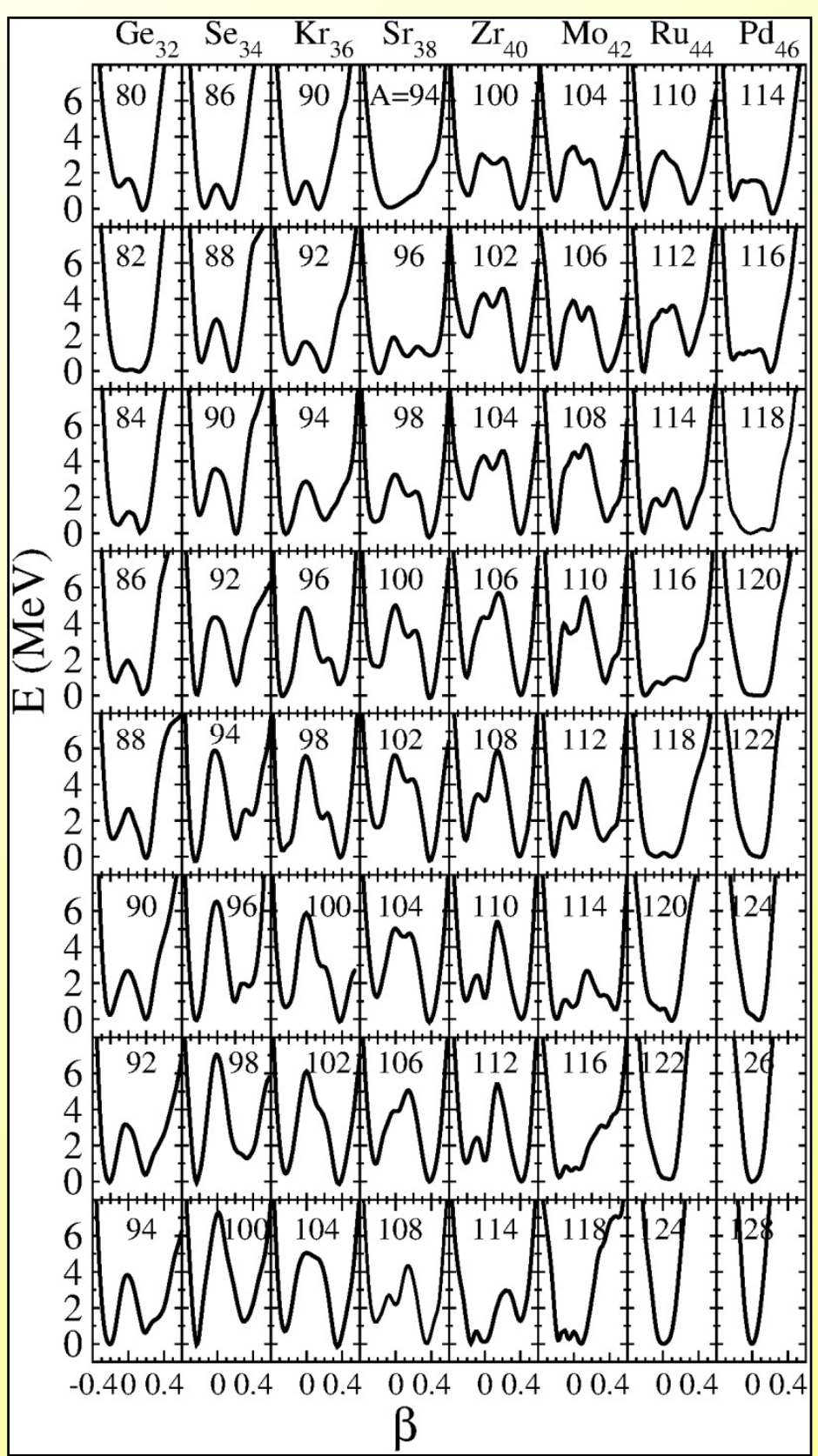




\section{$Z r-M_{0} B(G T)$}
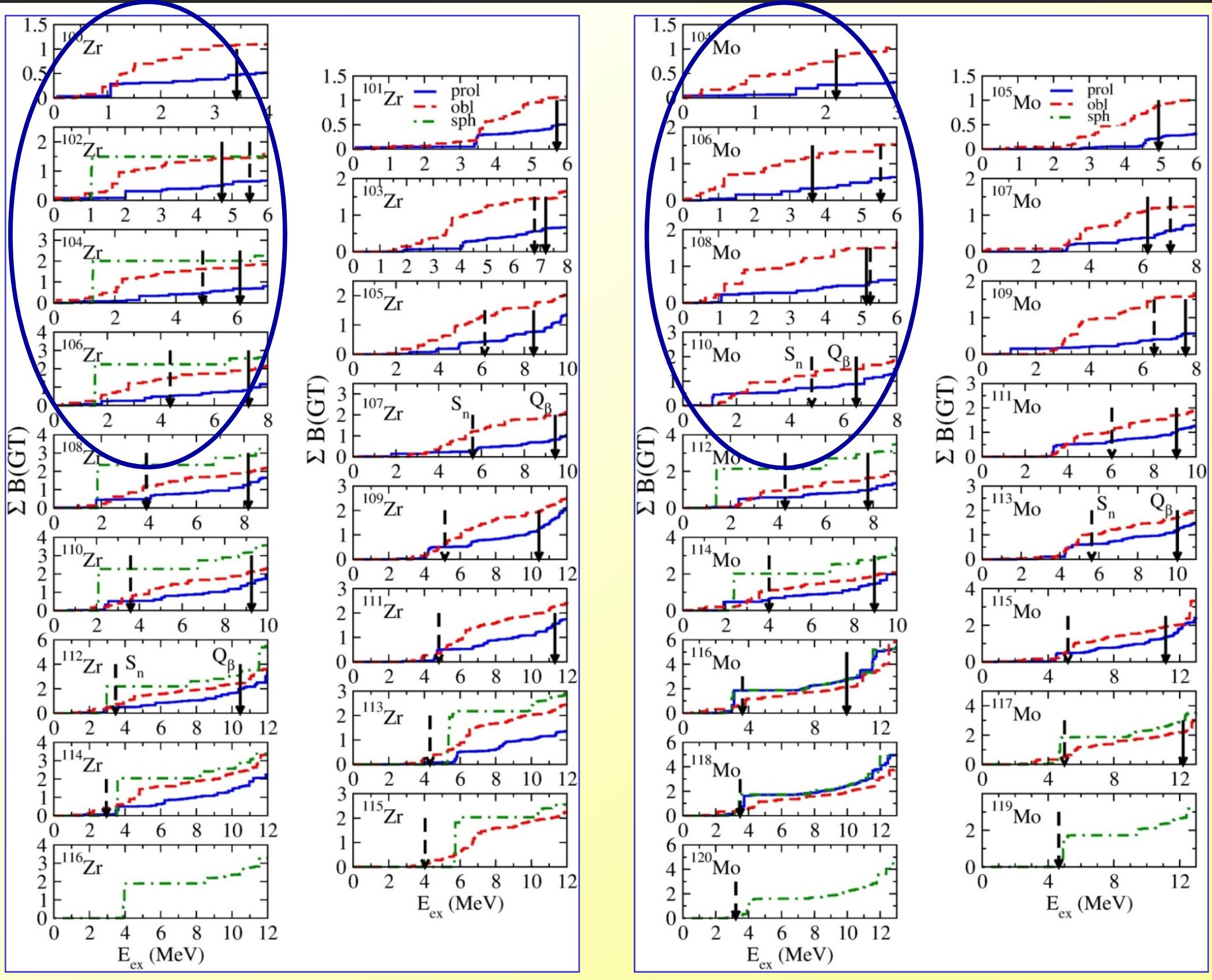


\section{$\mathrm{Zr}$-Mo Half-lives}
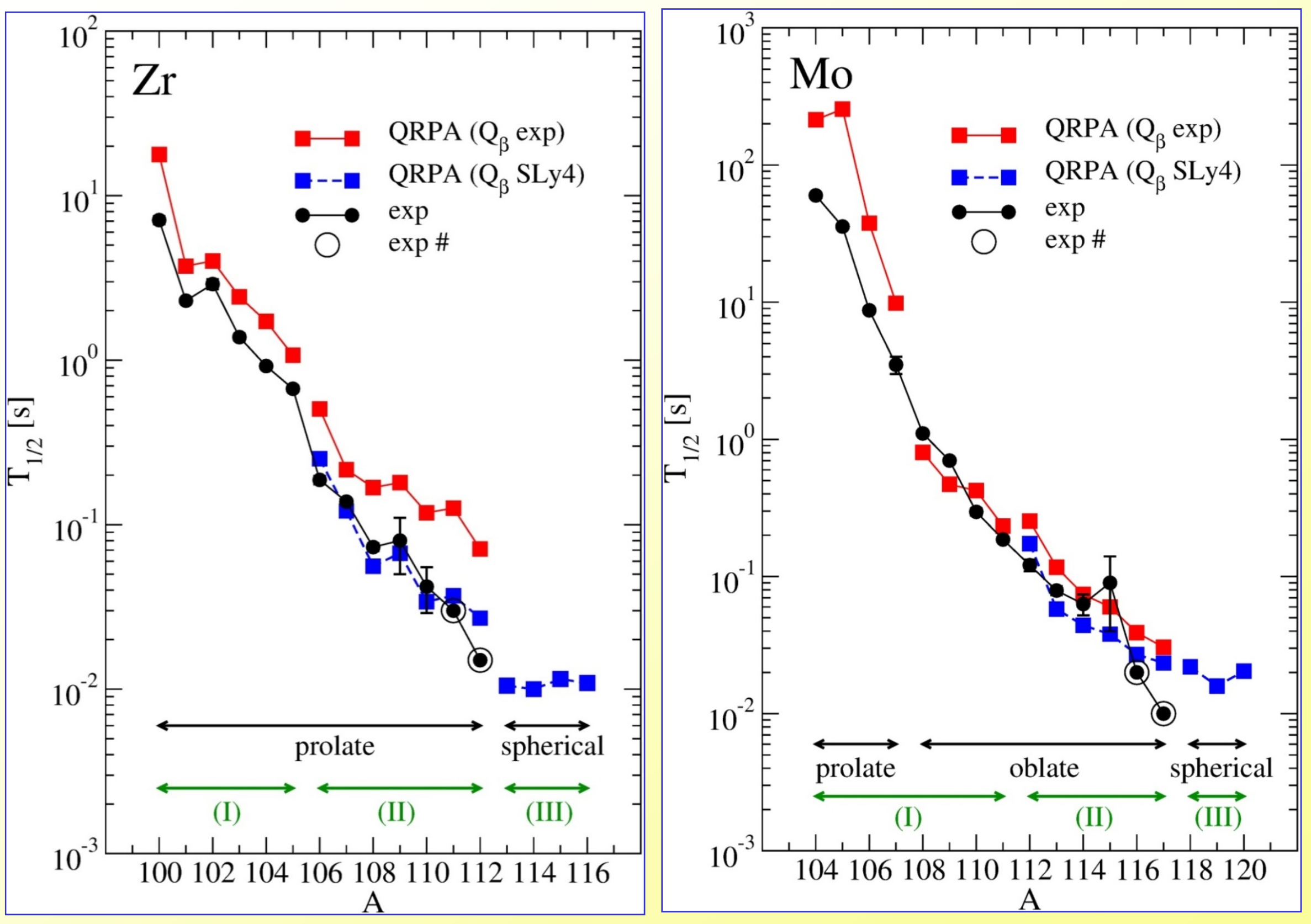


\section{Impact of deformation}
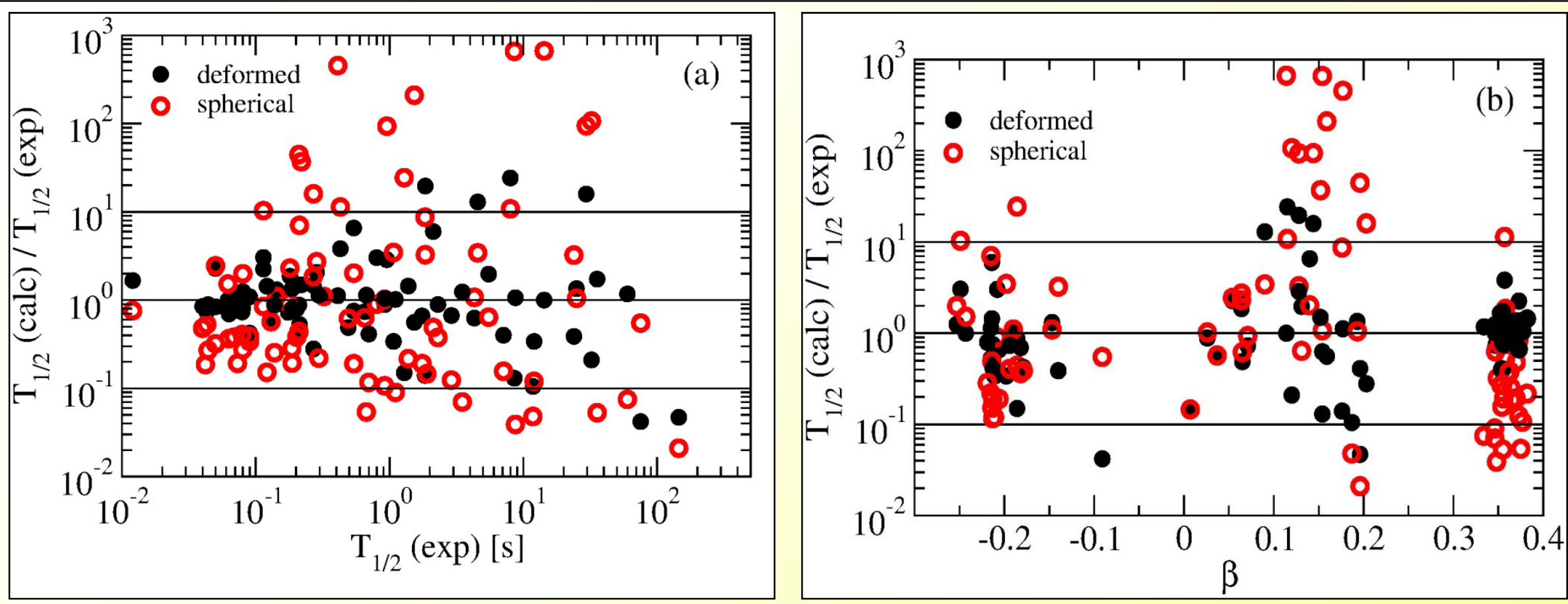

$$
r=\log _{10}\left[\frac{T_{1 / 2}(\text { calc })}{T_{1 / 2}(\exp )}\right]
$$

Average position

$$
M_{r}=\frac{1}{n} \sum_{i=1}^{n} r_{i}
$$

Mean square fluctuation

$$
\sigma_{r}=\left[\frac{1}{n} \sum_{i=1}^{n}\left(r_{i}-M_{r}\right)^{2}\right]^{1 / 2}
$$

\begin{tabular}{|l|c|c|c|}
\hline & & $10^{\mathrm{Mr}}$ & $10^{\text {or }}$ \\
\hline Global & Sph & $\mathbf{1 . 1 0 5}$ & $\mathbf{1 0 . 2 1 3}$ \\
\hline & def & $\mathbf{0 . 9 3 7}$ & 3.088 \\
\hline $0.25<\beta<4$ & sph & 0.260 & 3.285 \\
& def & 1.062 & 1.518 \\
\hline \multicolumn{2}{|c|}{ P.S. PRC 91, 044304 (2015) } \\
\hline
\end{tabular}




\section{Weak decay rates in pf-shell nuclei}

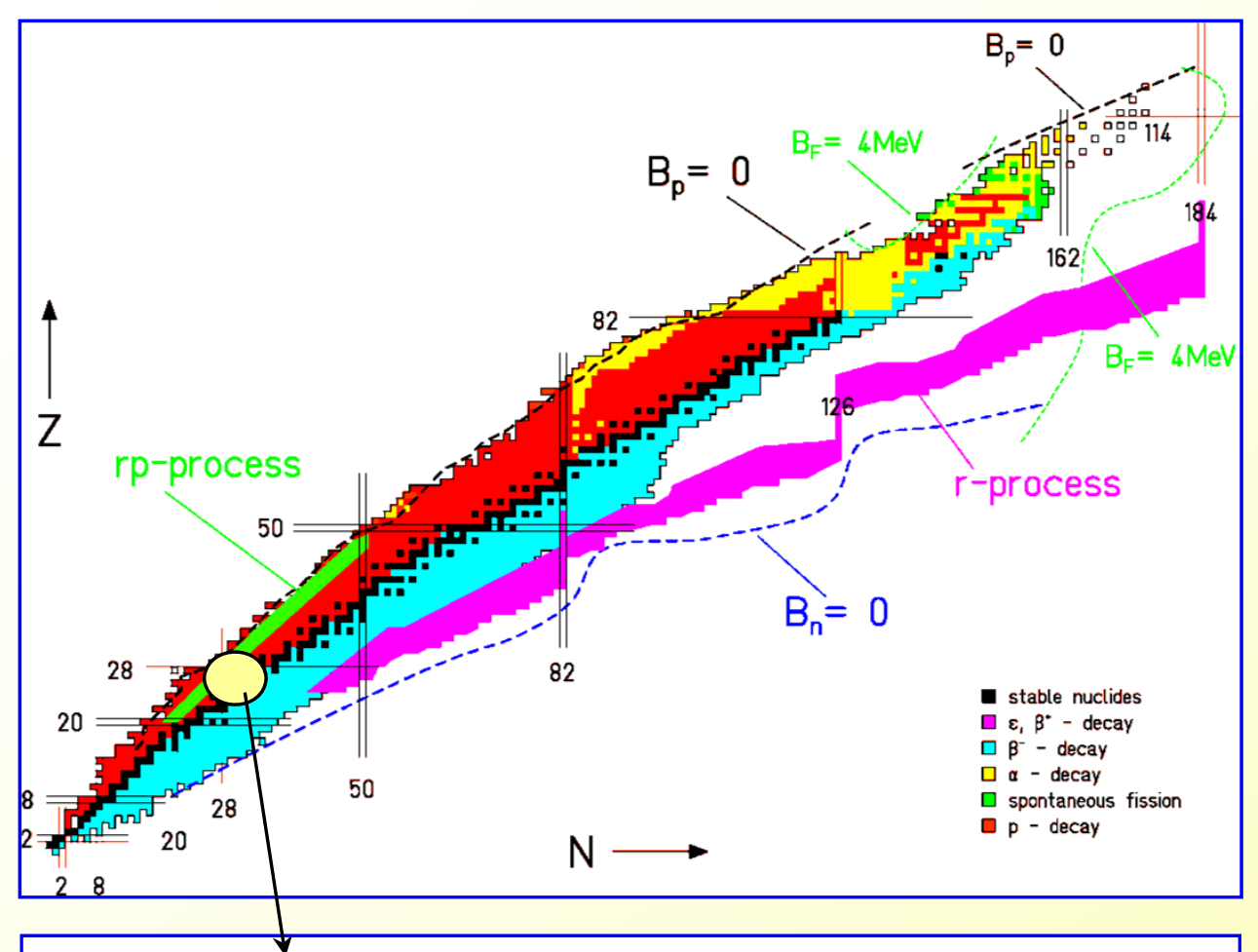

Main constituents of stellar core in presupernovae. Comparison with:

- $\exp .(n, p),(p, n)$

- SM calculations

GT properties: Test of QRPA

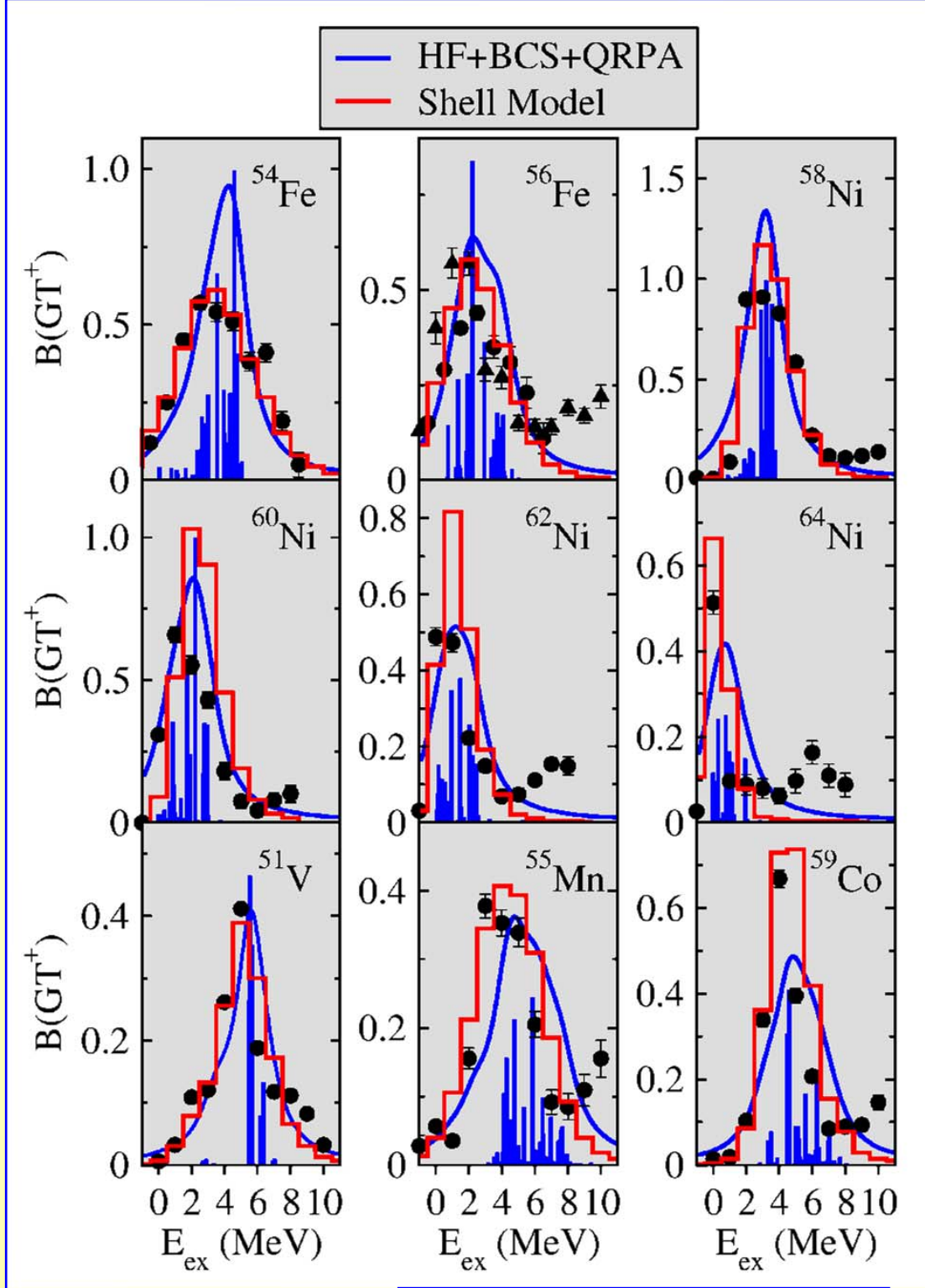

SM: NPA 653, 439 (1999) QRPA: NPA 716, 230 (2003) 


\section{Weak decay rates in pf-shell nuclei}

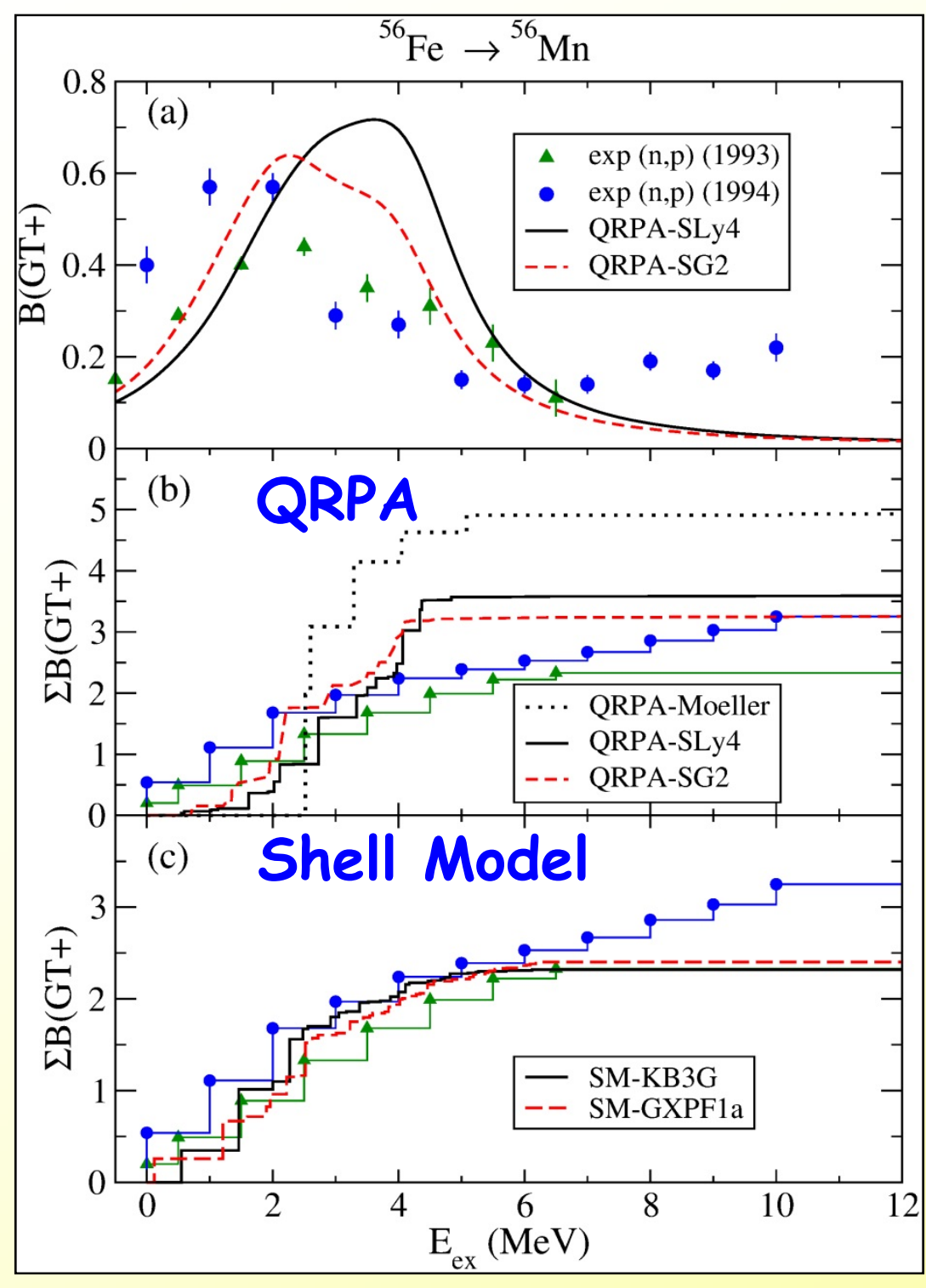

Calculations for several isotopes:

Sc, Ti, V, Fe, Mn, Ni, Co, Zn

exp: charge exchange reactions

$(n, p)$

$B_{i f}(G T) \propto\langle f|\sigma t| i\rangle^{2}$

Accumulated GT strength

A.L. Cole PRC 86, 015809 (2012)

P.S. PRC87, 045801 (2013), PRC93, 054309 (2016) 


\section{Weak decay rates in pf-shell nuclei}

$\lambda(\rho, T) \propto \sum_{i f} P_{i}(T) B_{i f} \Phi_{i f}(\rho, T)$

\section{Electron Capture from $e^{-}$plasma}

$$
\begin{array}{r}
\Phi_{i f}^{c E C}=\int_{\omega_{\ell}}^{\infty} \omega \sqrt{\omega^{2}-1}\left(Q_{i f}+\omega\right)^{2} F(Z, \omega) \times \\
S_{e}(\omega)\left[1-S_{v}\left(Q_{i f}+\omega\right)\right] d \omega
\end{array}
$$$$
S_{e}=\frac{1}{\exp \left[\left(\omega-\mu_{e}\right) /(k T)\right]+1}
$$

$Q_{i f}=\frac{1}{m_{e} c^{2}}\left(M_{p}-M_{d}+E_{i}-E_{f}\right)$

\section{Si burning}

\section{Ia Supernova}

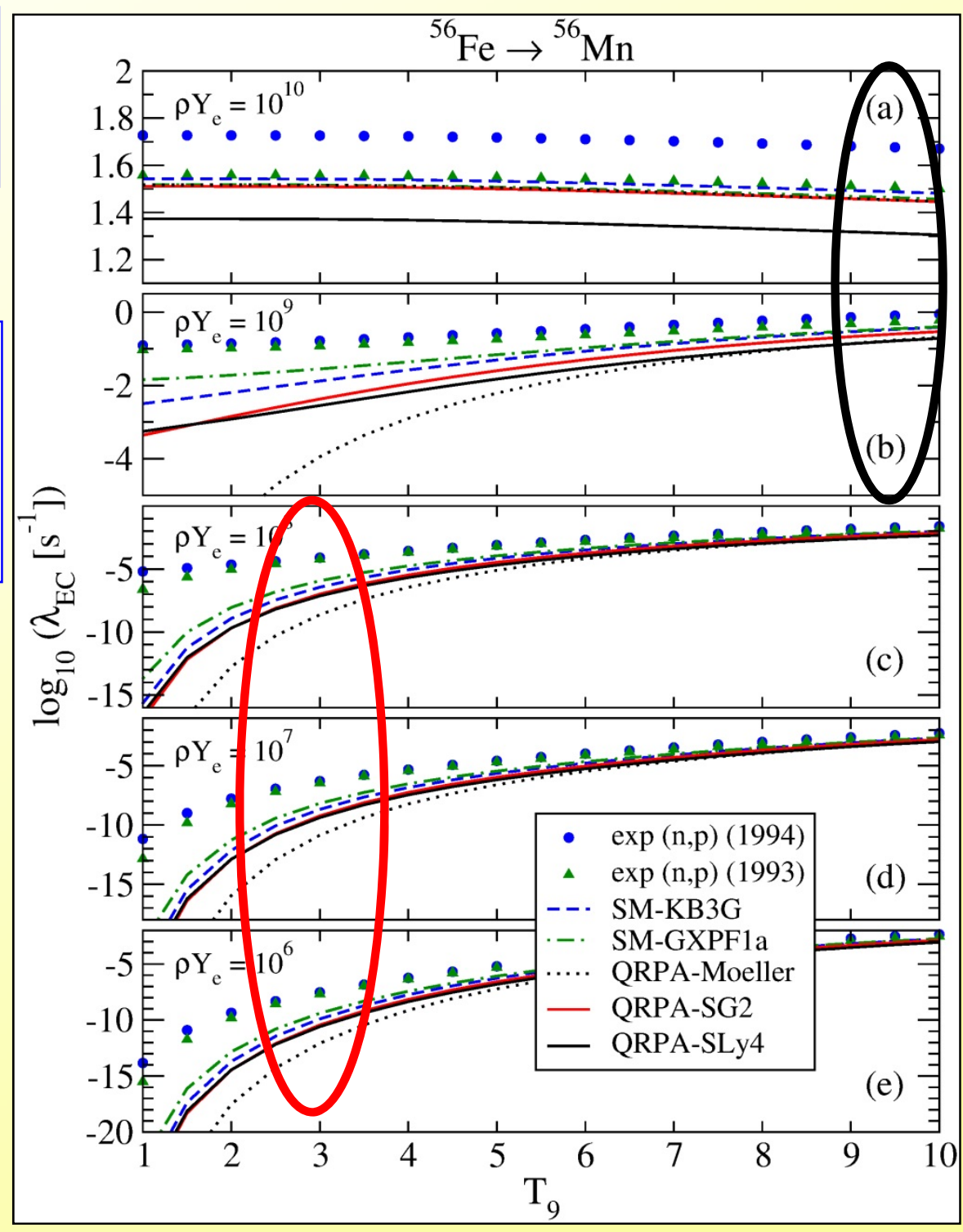




\section{Medium-mass neutron-deficient isotopes}

$X$-ray bursts: Source of intense $X$-ray emissions generated by thermonuclear runaway in the H-rich environment of an accreting $n$-star, fed from a binary red giant companion.

Nucleosynthesis mechanism: rp capture process

rp-process: Proton capture reaction rates are orders of magitude faster than the competing $\beta^{+}$-decays.

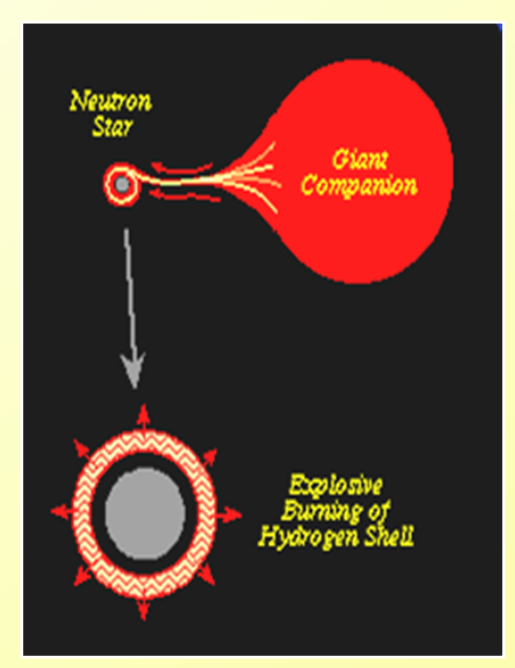

Waiting point nuclei: When the dominant $p$-capture is inhibited, the reaction flow waits for a slow beta-decay to proceed further.

Time scale

Isotope abundances

Light curve profile

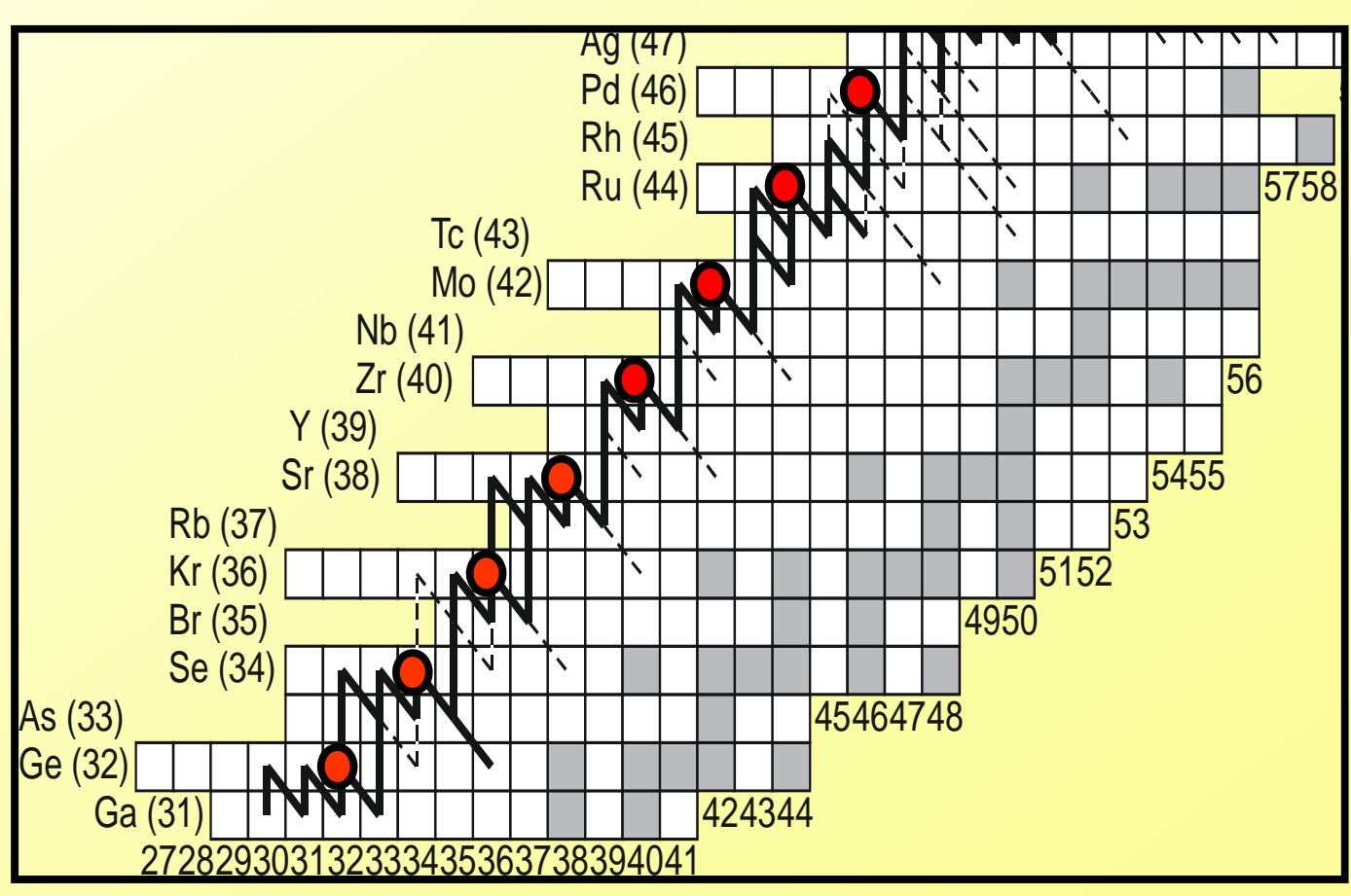




\section{Medium-mass neutron-deficient isotopes}

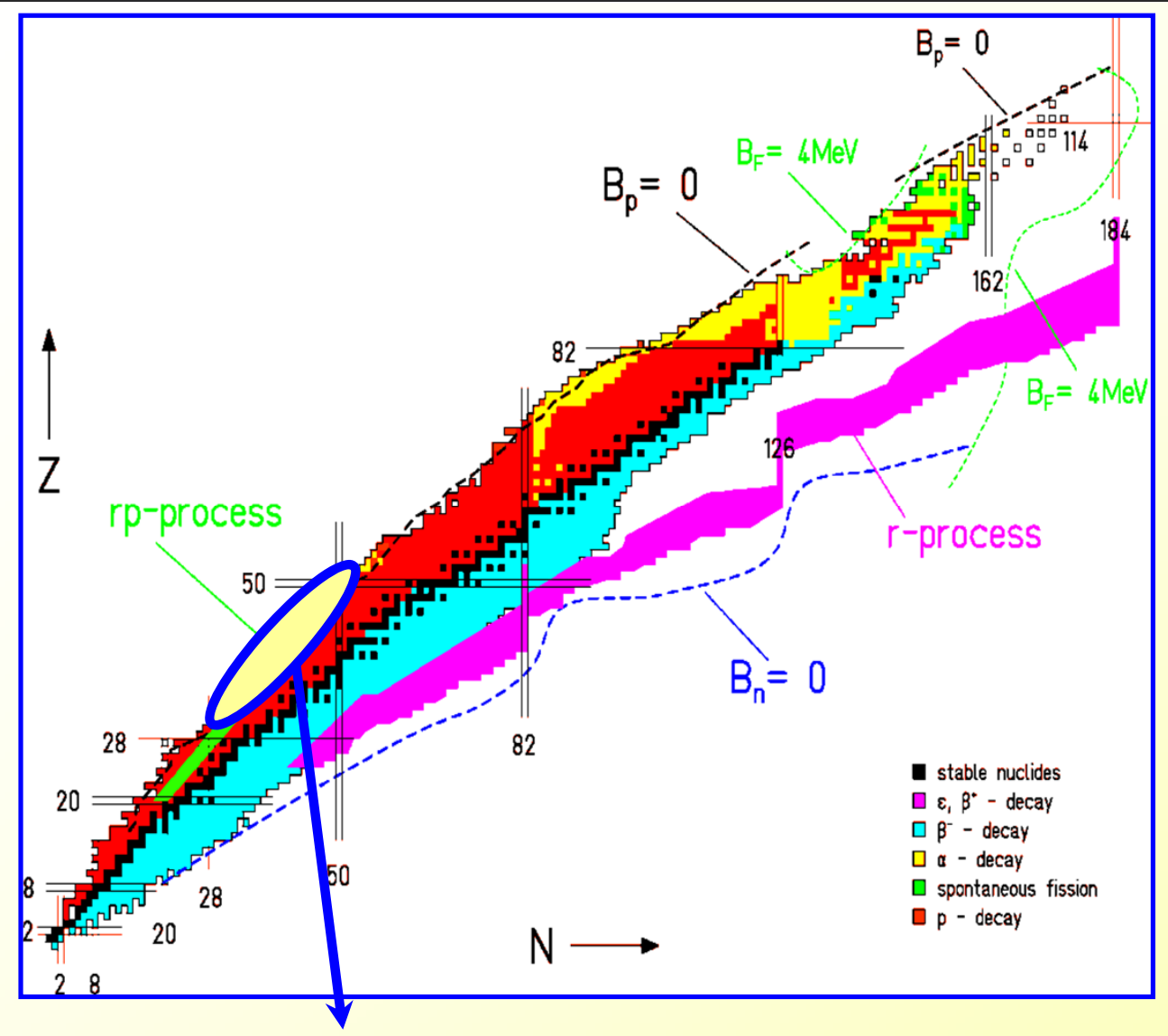

Waiting point nuclei in rp-processes

Shape coexistence

Beyond full Shell Model

$\mathrm{Ni}, \mathrm{Zn}, \mathrm{Ge}, \mathrm{Se}, \mathrm{Kr}, \mathrm{Sr}, \mathrm{Zr}, \mathrm{Mo}, \mathrm{Ru}, \mathrm{Pd}, \mathrm{Cd}, \mathrm{Sn}$ ( $N=Z$ and neighbors)

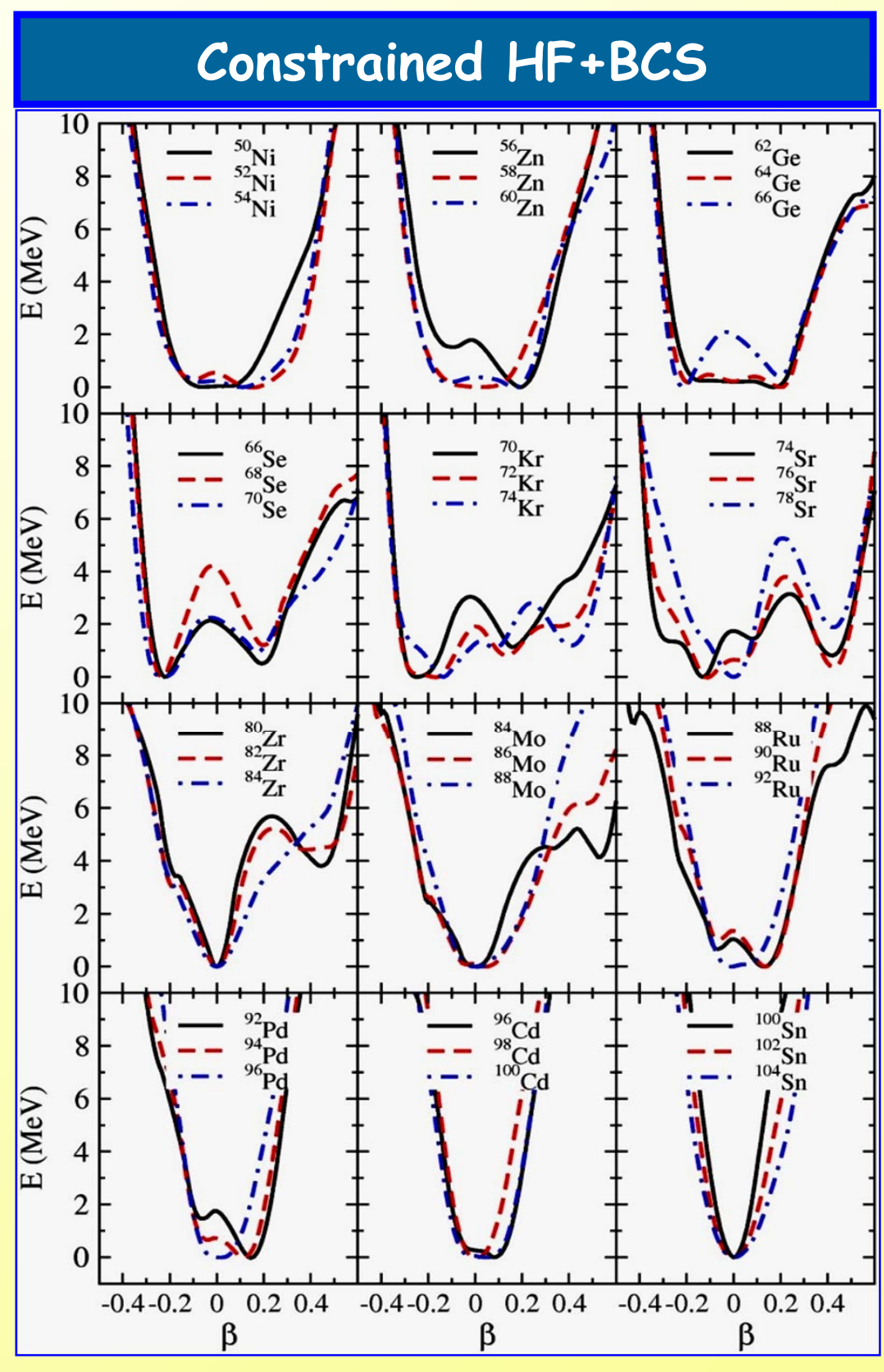




\section{Gamow-Teller strength: Theory and Experiment}

ISOLDE: Total absorption spectroscopy

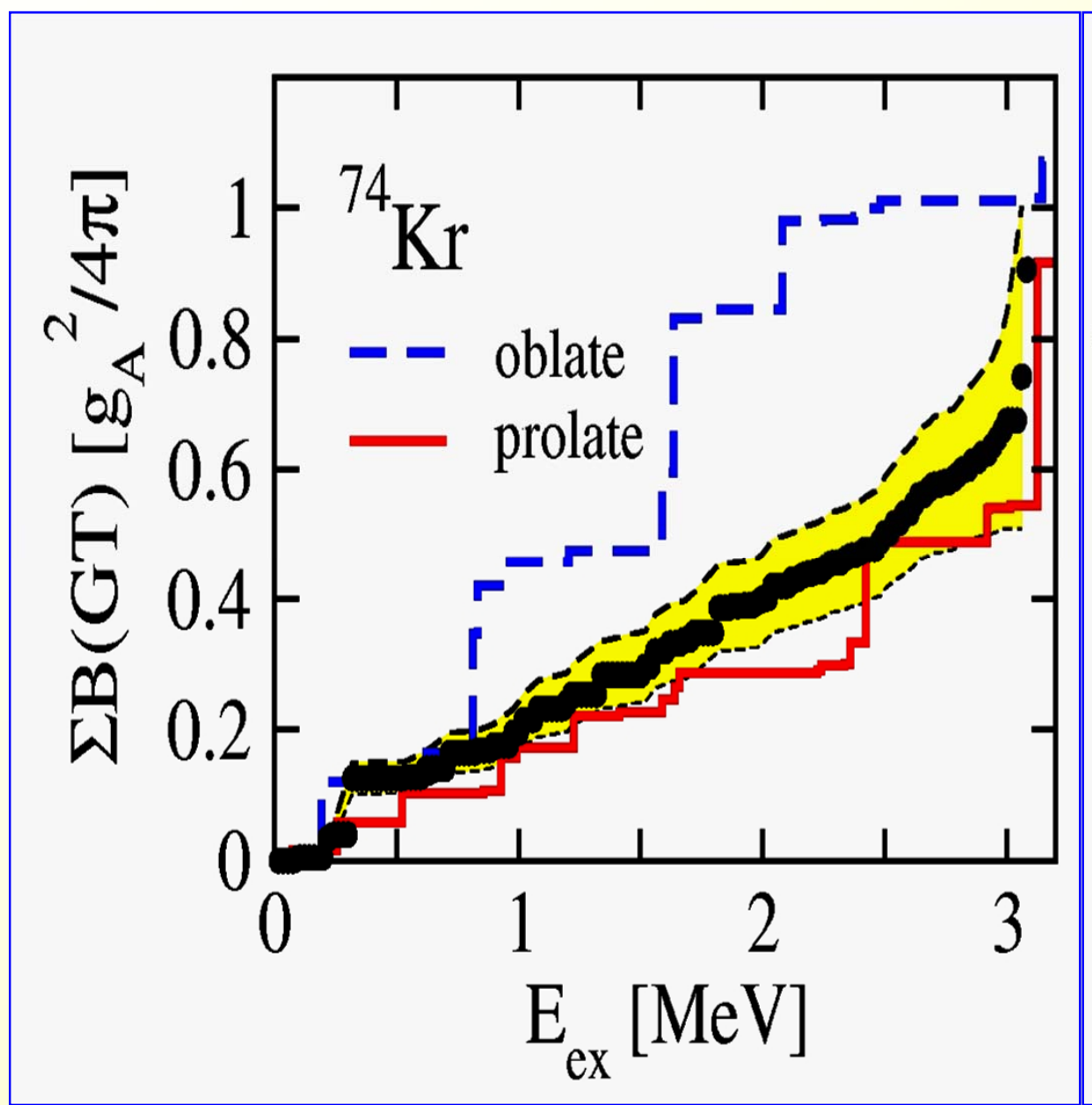

Exp: Poirier et al. PRC69, 034307 (2004)

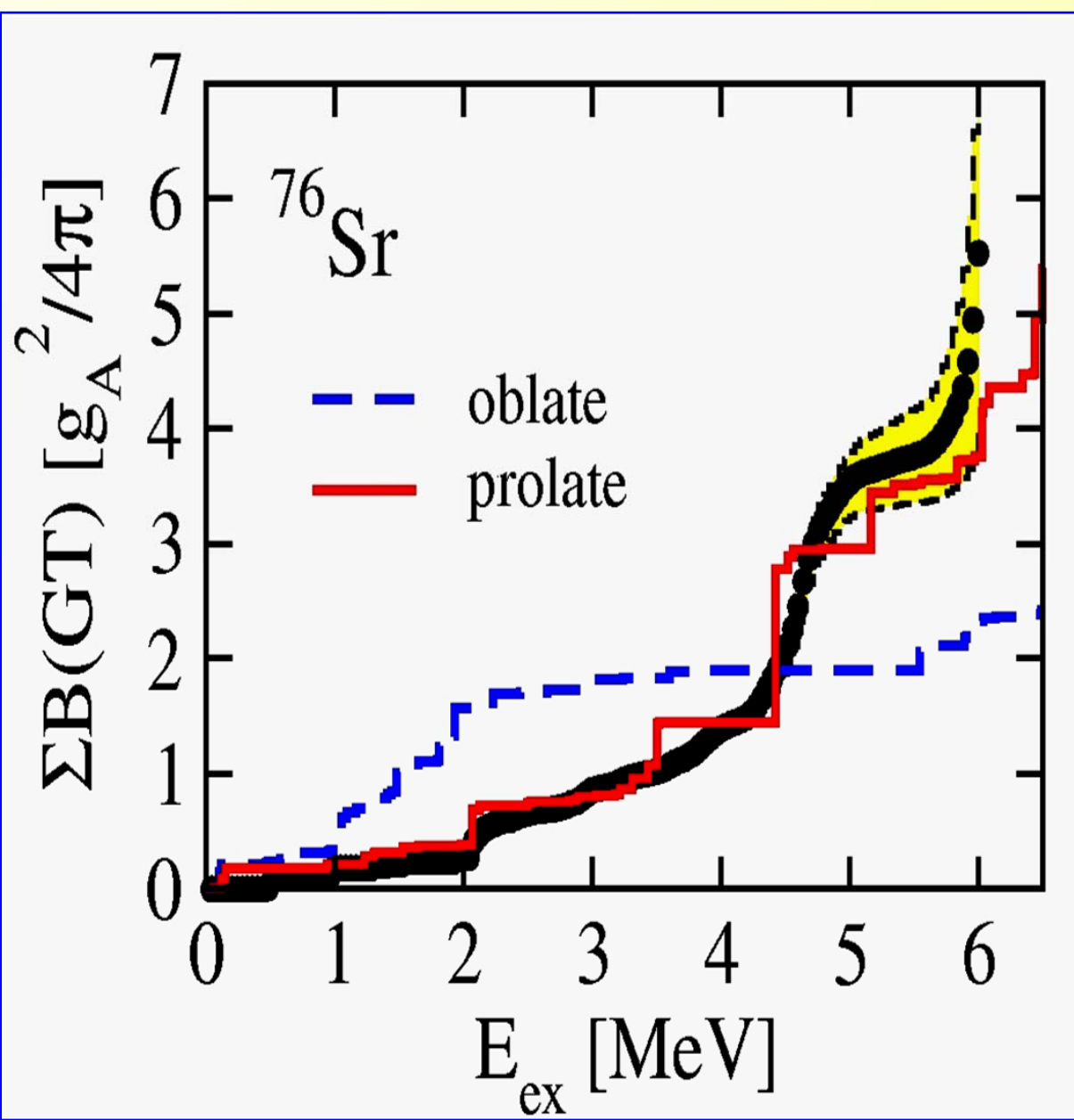

Exp: Nacher et al. PRL92, 232501 (2004) 


\section{$\beta^{+} /$EC half-lives: Theory and Experiment}

$$
T_{1 / 2}^{-1}=\frac{\left(g_{A} / g_{V}\right)_{\mathrm{eff}}^{2}}{6200} \sum_{f} \Phi^{\beta^{+} / E C}\left|\left\langle f\left\|\beta^{+}\right\| i\right\rangle\right|^{2}
$$$$
\Phi_{i f}^{\beta^{+}}=\int_{1}^{Q_{i f}} \omega \sqrt{\omega^{2}-1}\left(Q_{i f}-\omega\right)^{2} F(Z, \omega) d \omega
$$$$
\Phi^{E C}=\frac{\pi}{2}\left[q_{K}^{2} g_{K}^{2} B_{K}+q_{L_{1}}^{2} g_{L_{1}}^{2} B_{L_{1}}+\cdots\right]
$$

$$
\left(g_{A} / g_{V}\right)_{\text {eff }}=0.74\left(g_{A} / g_{V}\right)_{\text {bare }}
$$

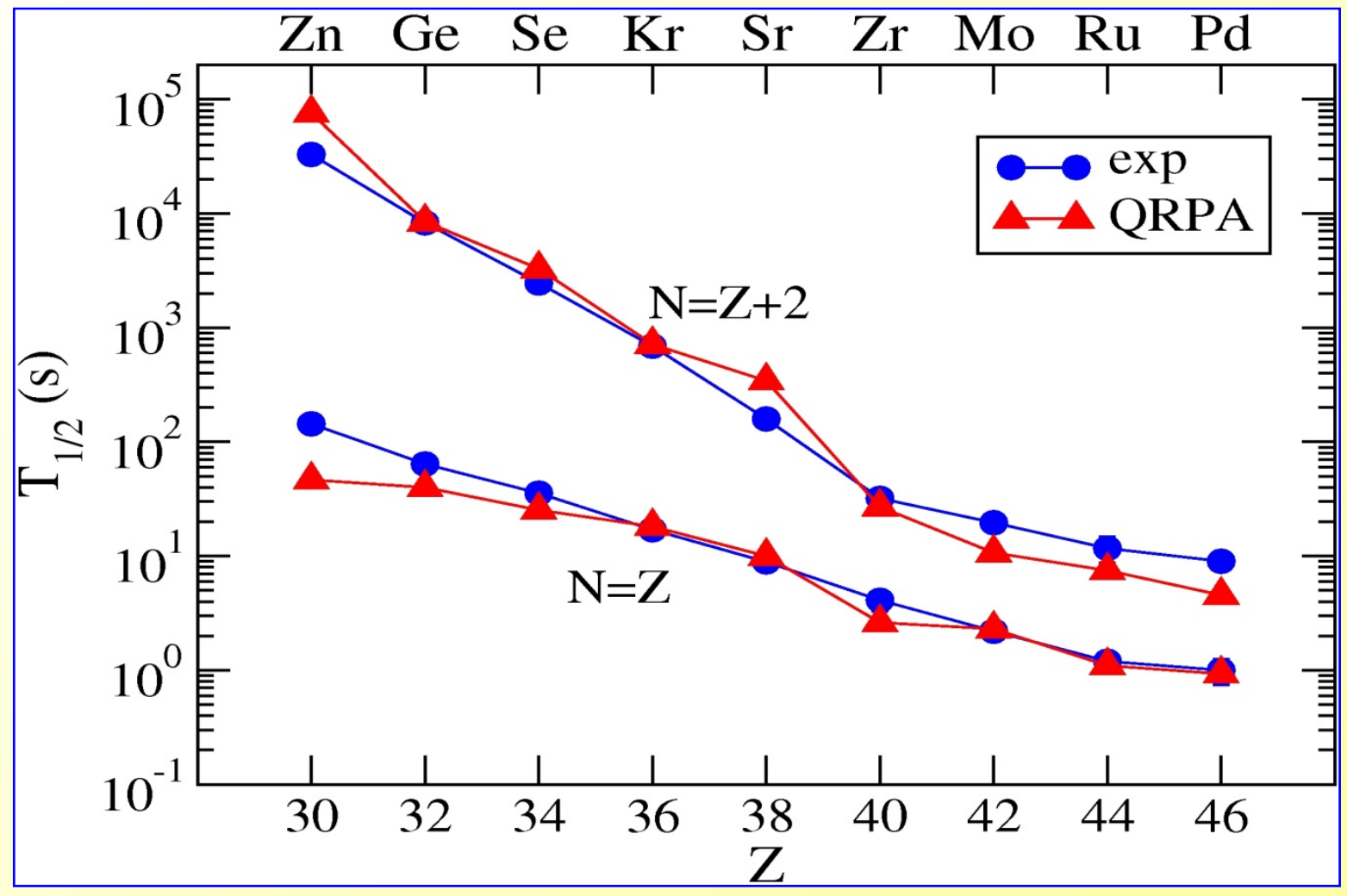

Good agreement with experiment:

Reliable extrapolations to high $\rho$ and $T$ 


\section{Weak decay rates in rp-process}

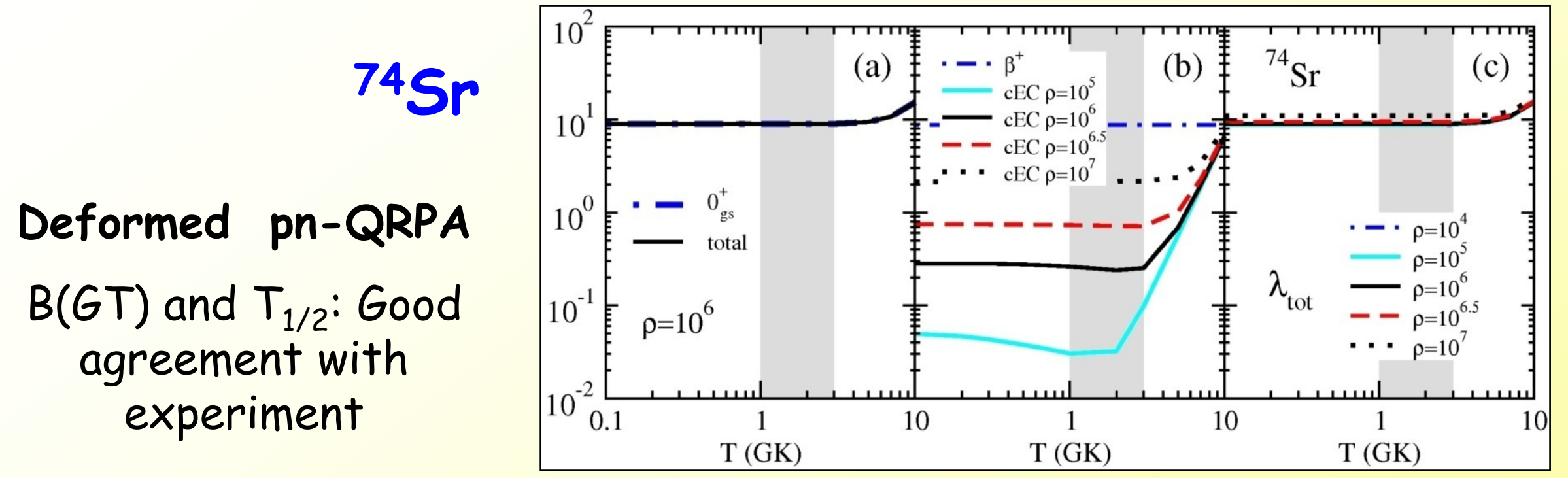

Competition $\beta+/ E C$

P.S. PRC83, 025801 (2011)

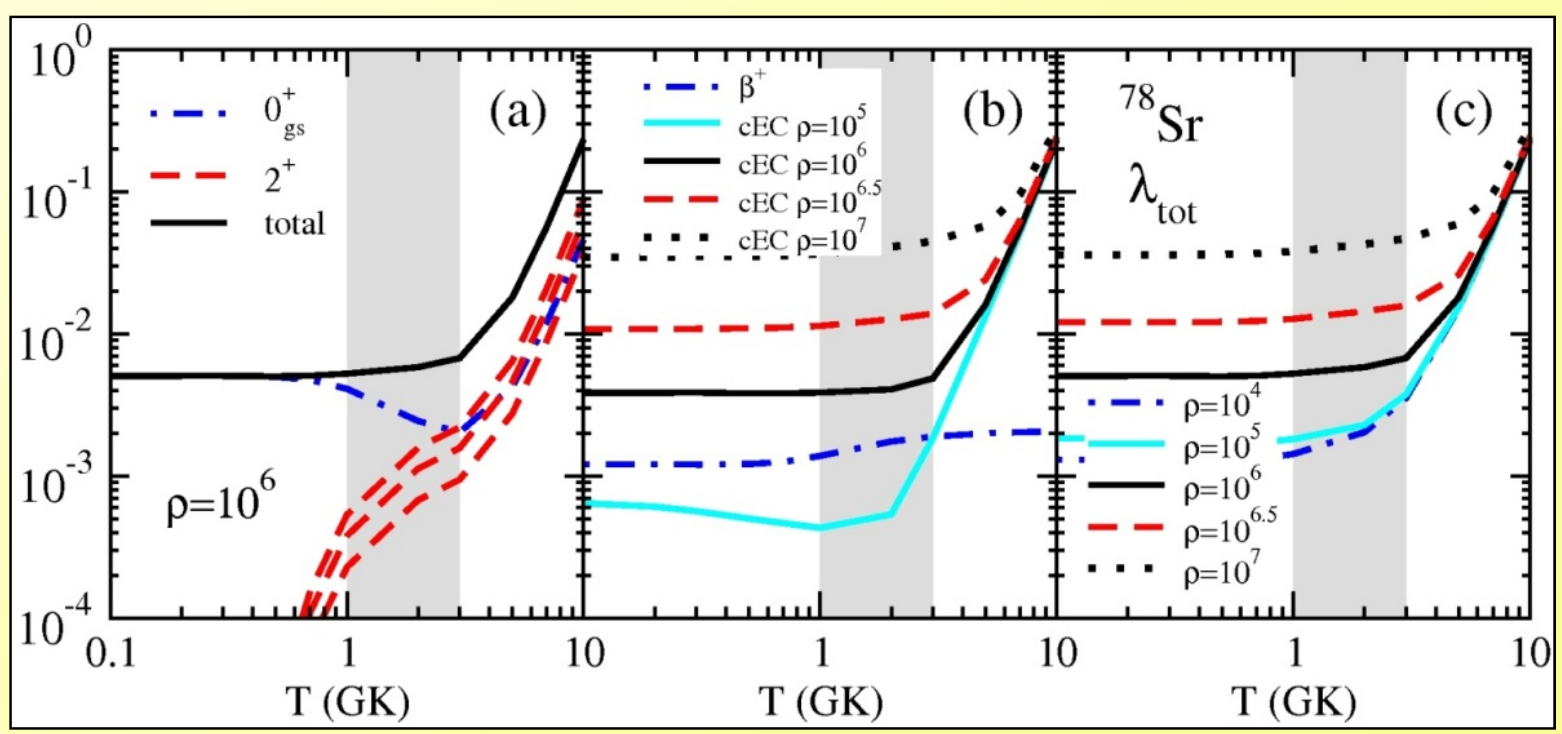

$78 \mathrm{Sr}$ 


\section{Weak decay rates in rp-process}

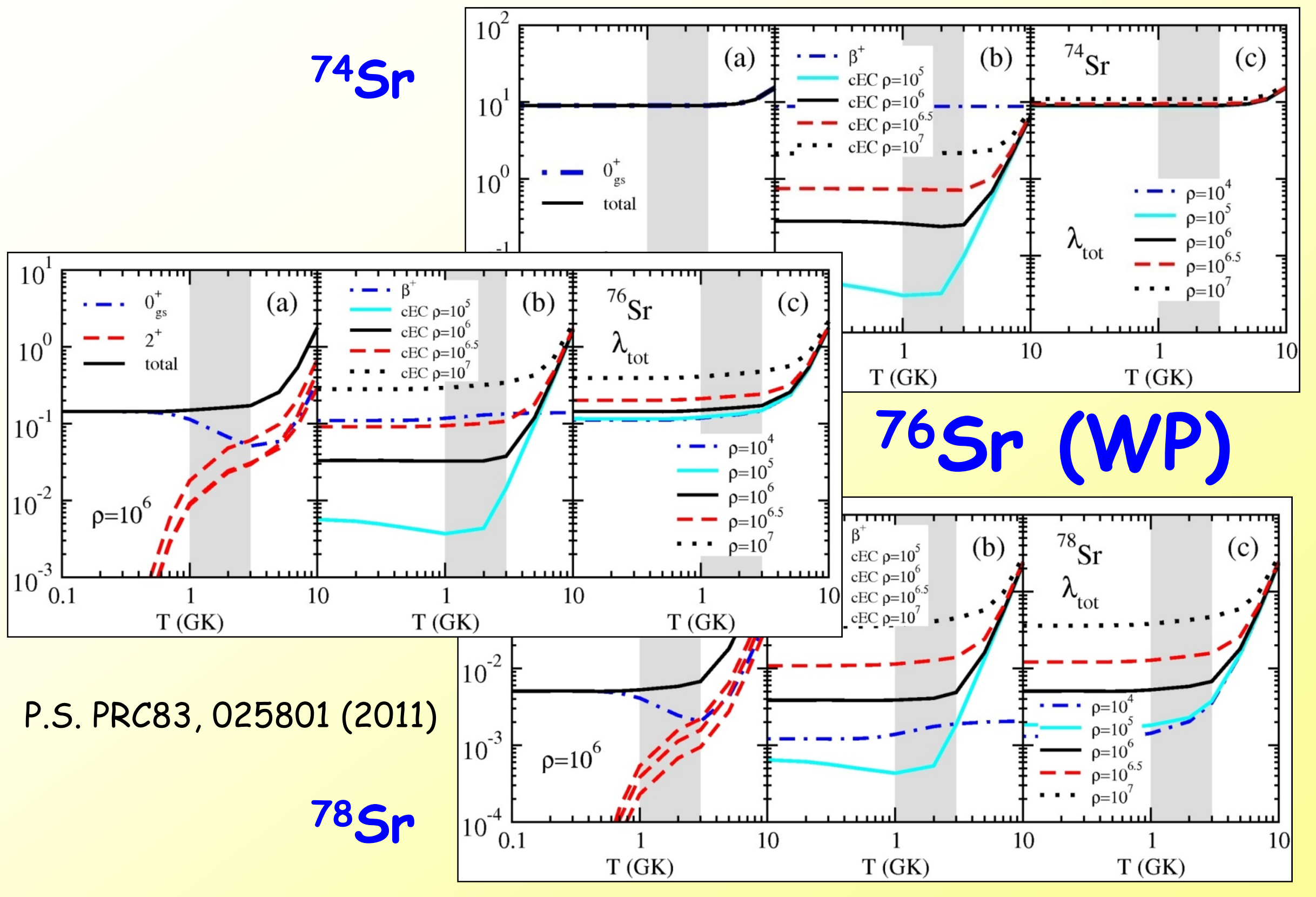




\section{Conclusions}

Nuclear structure model (deformed Skyrme HF+BCS+QRPA)

- Study of decay properties in different mass regions. Astrophysical applications.

- Reproduce half-lives and main features of GT strength distributions extracted from beta-decay and/or charge exchange reactions.

- Weak-decay rates at $(\rho, T)$ typical of astrophysical scenarios:

$>$ pf-shell nuclei (presupernova): EC rates from QRPA comparable quality to benchmark SM calculations.

$>$ Neutron-rich $\mathrm{Zr}$-Mo isotopes ( $r$ process).

$>$ Neutron-deficient WP nuclei (rp-process):

$E C / \beta^{+}$competition 


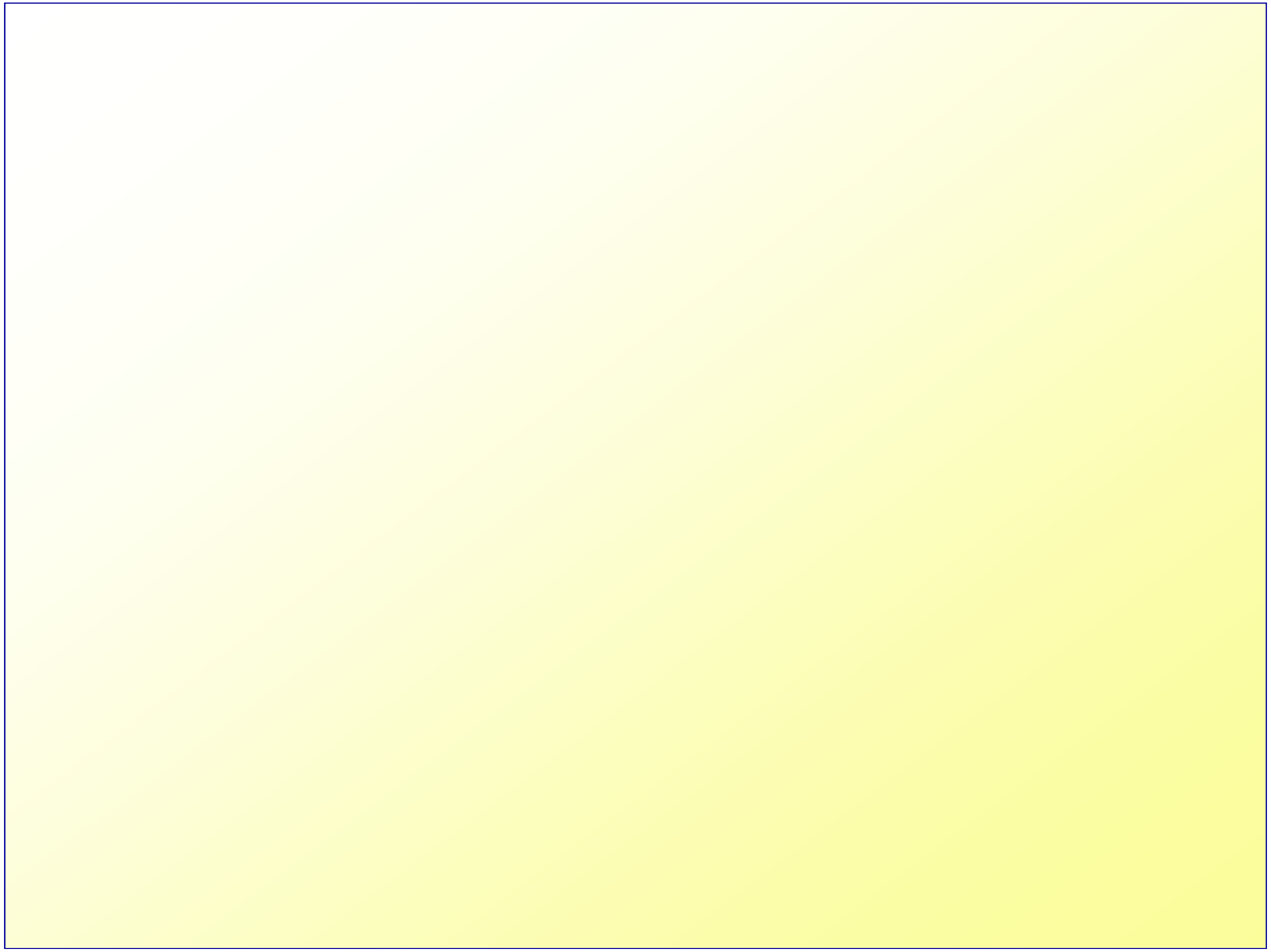




\section{Residual interactions}

\section{Particle-hole residual interaction consistent with the HF mean field}

$$
\begin{gathered}
V_{p h}=\frac{1}{16} \sum_{s t s^{\prime} t^{\prime}}\left[1+(-1)^{s-s^{\prime}} \overrightarrow{\sigma_{1}} \cdot \overrightarrow{\sigma_{2}}\right]\left[1+(-1)^{t-t^{\prime}} \overrightarrow{\tau_{1}} \cdot \overrightarrow{\tau_{2}}\right] \frac{\delta^{2} E}{\delta \rho_{s t}\left(\overrightarrow{r_{1}}\right) \delta \rho_{s^{\prime} t^{\prime}}\left(\overrightarrow{r_{2}}\right)} \delta\left(\overrightarrow{r_{1}}-\overrightarrow{r_{2}}\right) \\
V_{p h}^{\sigma \tau}=\frac{1}{16} \sum_{s t s^{\prime} t^{\prime}}(-1)^{s-s^{\prime}}(-1)^{t-t^{\prime}} \overrightarrow{\sigma_{1}} \cdot \overrightarrow{\sigma_{2}} \overrightarrow{\tau_{1}} \cdot \overrightarrow{\tau_{2}} \frac{\delta^{2} E}{\delta \rho_{s t}\left(\overrightarrow{r_{1}}\right) \delta \rho_{s^{\prime} t^{\prime}}\left(\overrightarrow{r_{2}}\right)} \delta\left(\overrightarrow{r_{1}}-\overrightarrow{r_{2}}\right) \\
=\frac{1}{16}\left[-4 t_{0}-2 t_{1} k_{F}^{2}+2 t_{2} k_{F}^{2}-\frac{2}{3} t_{3} \rho^{\alpha}\right] \overrightarrow{\sigma_{1}} \cdot \overrightarrow{\sigma_{2}} \overrightarrow{\tau_{1}} \cdot \overrightarrow{\tau_{2}} \delta\left(\overrightarrow{r_{1}}-\overrightarrow{r_{2}}\right)
\end{gathered}
$$

\section{Average over nuclear volume $\rightarrow$ Separable forces}

$$
\left\{\begin{array}{r}
V_{G T}^{p h}=2 \chi_{G T}^{p h} \sum_{K=0, \pm 1}(-1)^{K} \beta_{K}^{+} \beta_{-K}^{-}, \quad \beta_{K}^{+}=\sigma_{K} t^{+}=\sum_{\pi v}\left\langle v\left|\sigma_{K}\right| \pi\right\rangle a_{v}^{+} a_{\pi} \\
\chi_{G T}^{p h}=-\frac{3}{8 \pi R^{3}}\left\{t_{0}+\frac{1}{2} k_{F}^{2}\left(t_{1}-t_{2}\right)+\frac{1}{6} t_{3} \rho^{\alpha}\right\} \\
V_{G T}^{p p}=-2 \kappa_{G T}^{p p} \sum_{K=0, \pm 1}(-1)^{K} P_{K}^{+} P_{K}, \quad P_{K}^{+}=\sum_{\pi v}\left\langle v\left|\left(\sigma_{K}\right)^{+}\right| \pi\right\rangle a_{v}^{+} a_{\pi}^{+}
\end{array}\right.
$$




\section{PNQRPA with separable forces}

Phonon operator

$$
\begin{array}{r}
\Gamma_{\omega_{K}}^{+}=\sum_{\gamma_{K}}\left[X_{\gamma_{K}}^{\omega_{K}} A_{\gamma_{K}}^{+}-Y_{\gamma_{K}}^{\omega_{K}} A_{\gamma_{K}}\right], \quad A_{\gamma_{K}}^{+}=\alpha_{n}^{+} \alpha_{\bar{p}}^{+} \\
\Gamma_{\omega_{K}}|0\rangle=0, \quad \Gamma_{\omega_{K}}^{+}|0\rangle=\left|\omega_{K}\right\rangle
\end{array}
$$

pnQRPA equations

$$
\text { Transition amplitudes }\left\langle\omega_{K}\left|\sigma_{K} t^{ \pm}\right| 0\right\rangle=\mp M_{ \pm}^{\omega_{K}}
$$

$$
\begin{aligned}
& M_{-}^{\omega_{K}}=\sum_{\pi v}\left(q_{\pi v} X_{\pi v}^{\omega_{K}}+\tilde{q}_{\pi v} Y_{\pi v}^{\omega_{K}}\right) M_{+}^{\omega_{K}}=\sum_{\pi v}\left(\tilde{q}_{\pi v} X_{\pi v}^{\omega_{K}}+q_{\pi v} Y_{\pi v}^{\omega_{K}}\right) \\
& \tilde{q}_{\pi v}=u_{v} v_{\pi} \Sigma_{K}^{\pi v}, \quad q_{\pi v}=v_{v} u_{\pi} \sum_{K}^{\pi v}, \quad \sum_{K}^{\pi v}=\left\langle v\left|\sigma_{K}\right| \pi\right\rangle
\end{aligned}
$$

Single-particle states from deformed Skyrme Hartree-Fock u, v: Occupation amplitudes from BCS pairing

$$
\begin{array}{|l|}
\hline \mathrm{B}(G T) \text { in the lab. system } \quad I_{i} K_{i}\left(0^{+} 0\right) \rightarrow I_{f} K_{f}\left(1^{+} K\right) \\
\hline B_{\omega}\left(G T^{ \pm}\right)=\delta\left(\omega_{0}-\omega\right)\left\langle\omega_{0}\left|\sigma_{0} t^{ \pm}\right| 0\right\rangle^{2}+2 \delta\left(\omega_{1}-\omega\right)\left\langle\omega_{1}\left|\sigma_{1} t^{ \pm}\right| 0\right\rangle^{2} \\
\hline
\end{array}
$$




\section{Isotopic evolution $\left(\beta, r_{c}\right)$ : Ge-Pd $n$-rich isotopes}
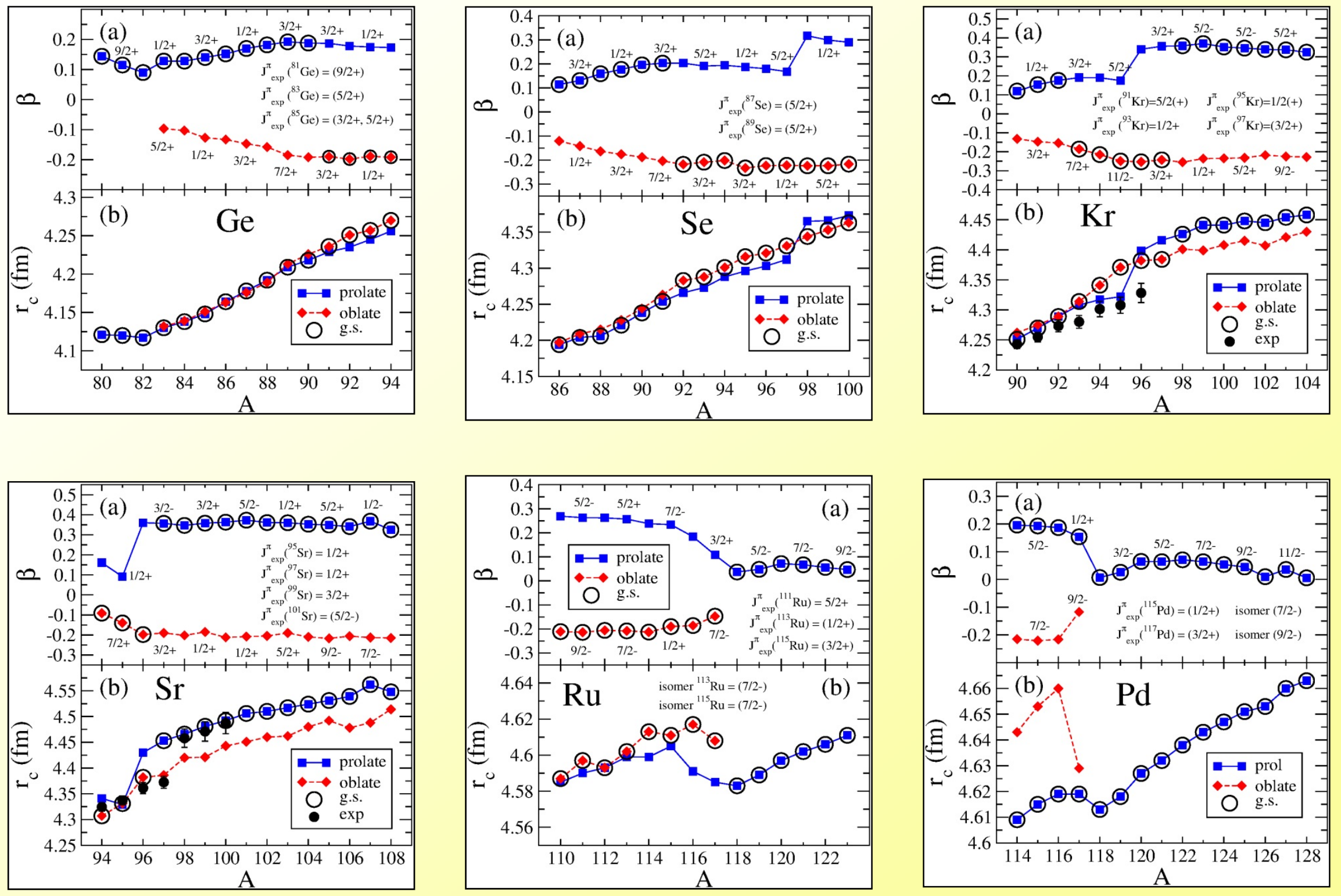


\section{Shape dependence of GTdistributions in neutron-deficient: Pb isotopes}

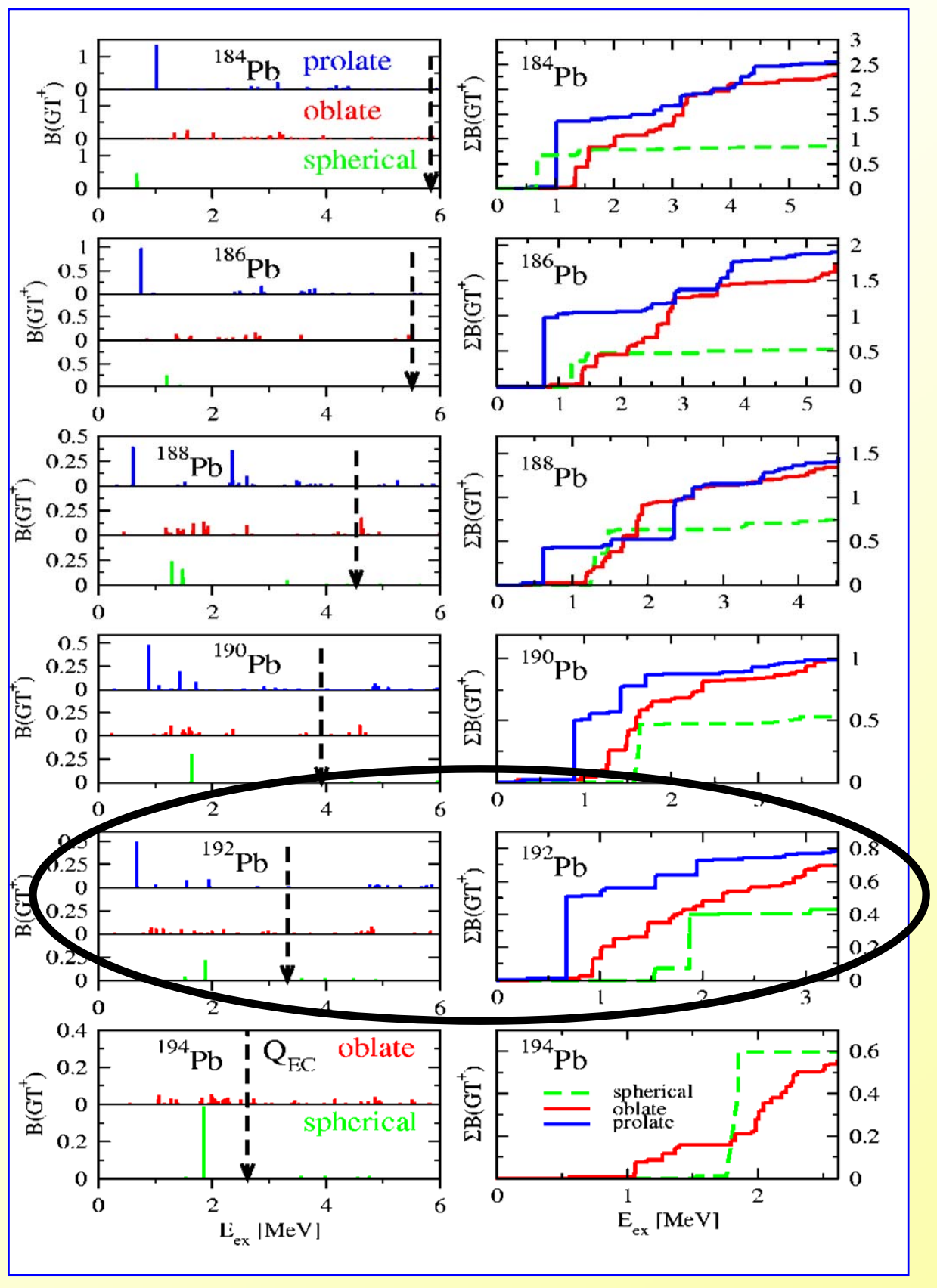

$B(G T)$ strength distributions

- Not very sensitive to : Skyrme force and pairing treatment

- Sensitive to: Nuclear shape

Signatures of deformation

PRC 72, 054317 (2005), PRC 73, 054302 (2006)

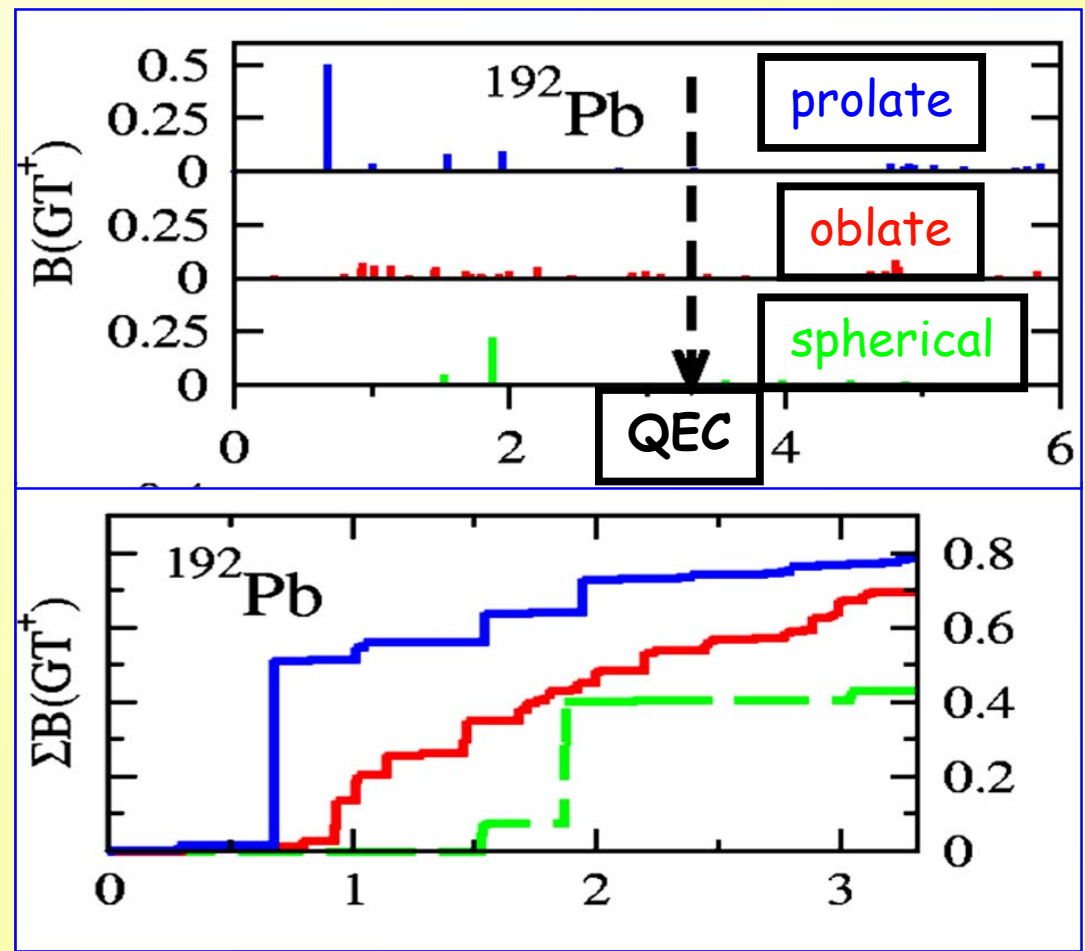




\section{$\beta$-decay patterns: Theory vs. Experiment}

$192 \mathrm{~Pb}$

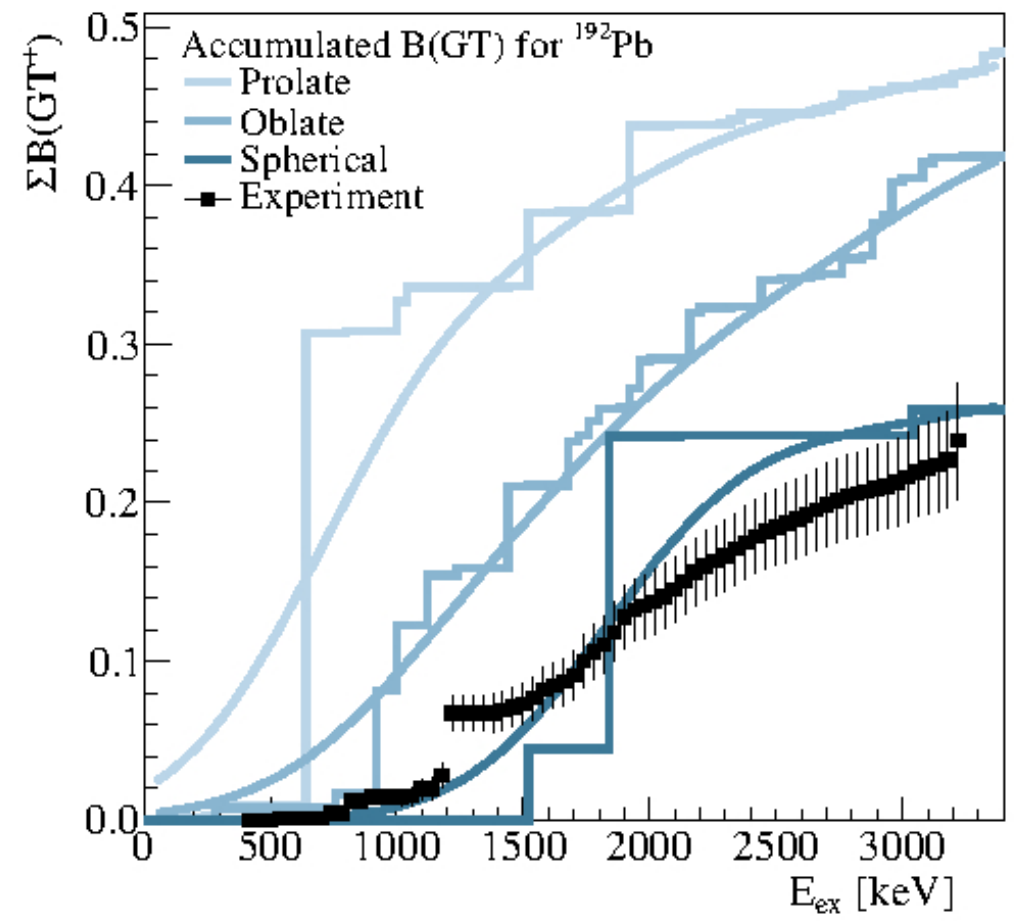

$190 \mathrm{~Pb}$

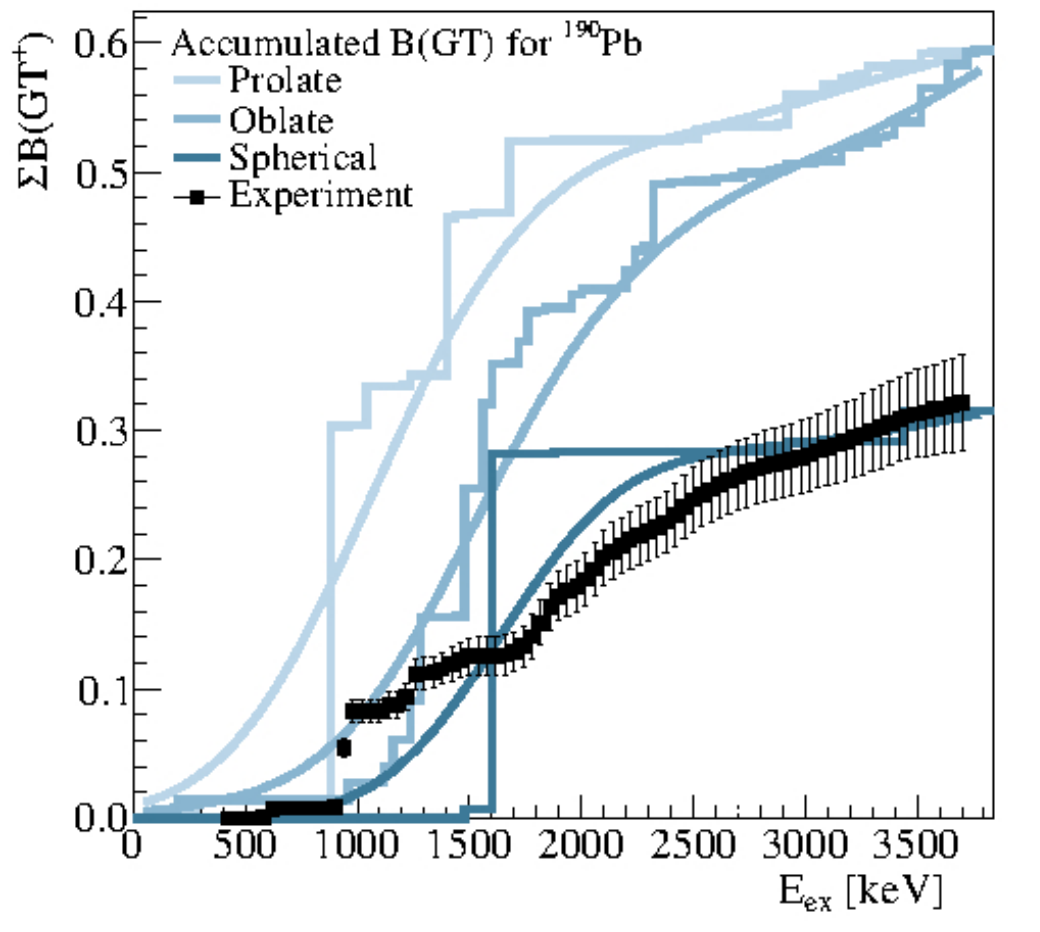

Preliminary experimental data (unpublished)

TAS (ISOLDE/CERN) A.Algora et al. 


\section{Weak decay rates in pf-shell nuclei}
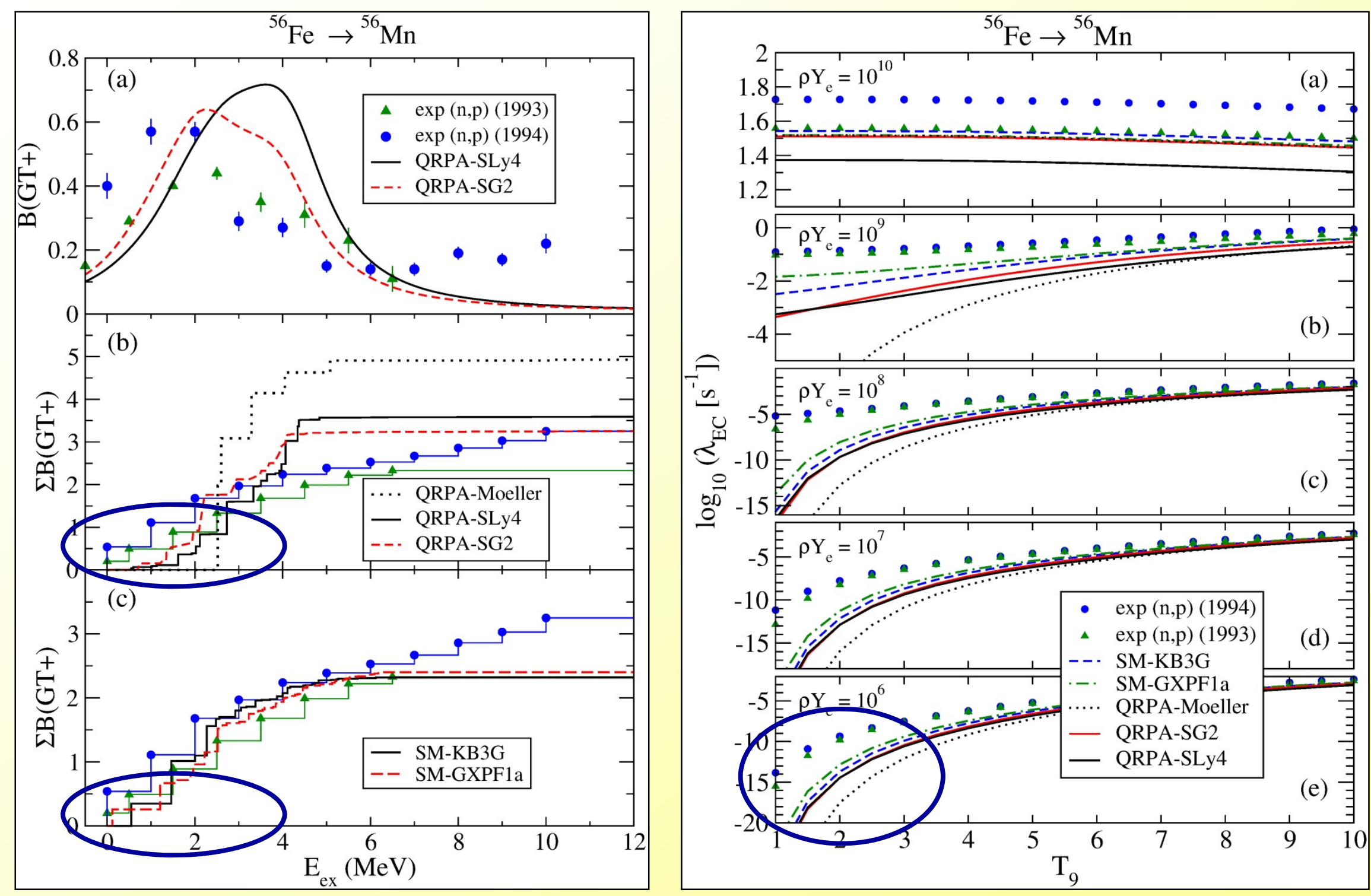

$(Q<0)$ large: sensitive low-lying exc.

P.S. PRC87, 045801 (2013) 


\section{Weak decay rates in pf-shell nuclei}
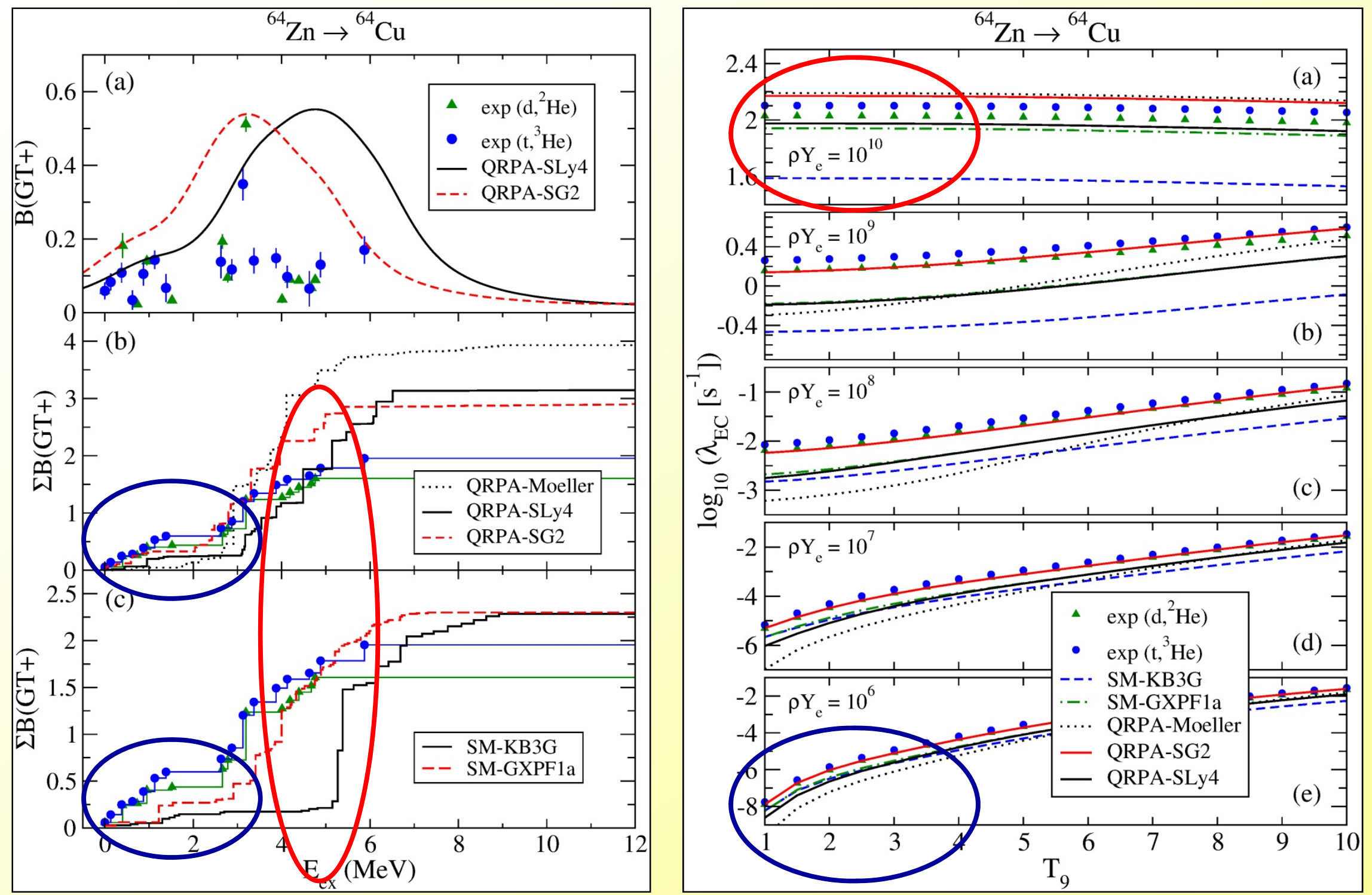

\section{$Q$ small}




\section{Odd-A nuclei. Excited states}

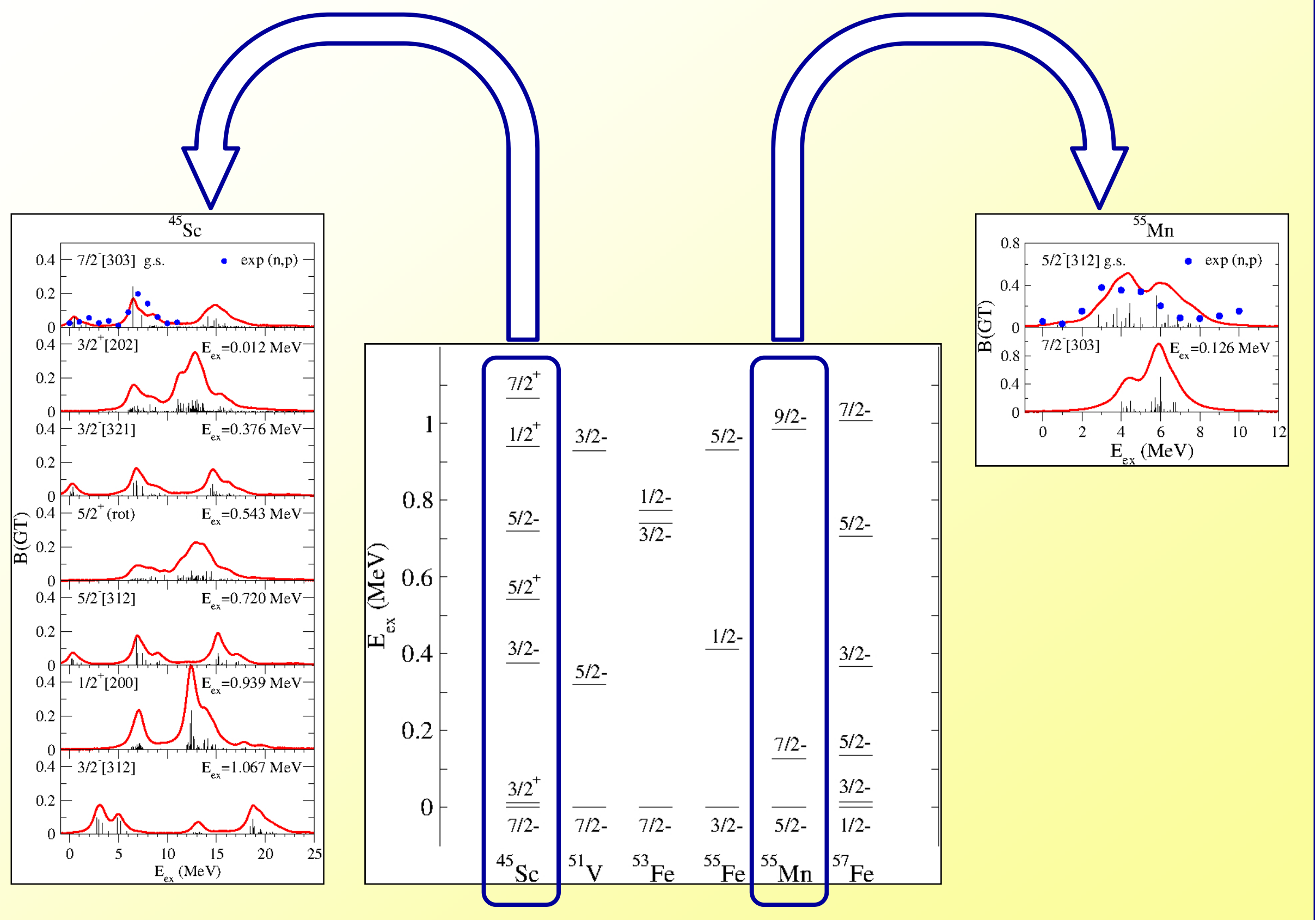




\section{Odd-A nuclei: Contributions of excited states}
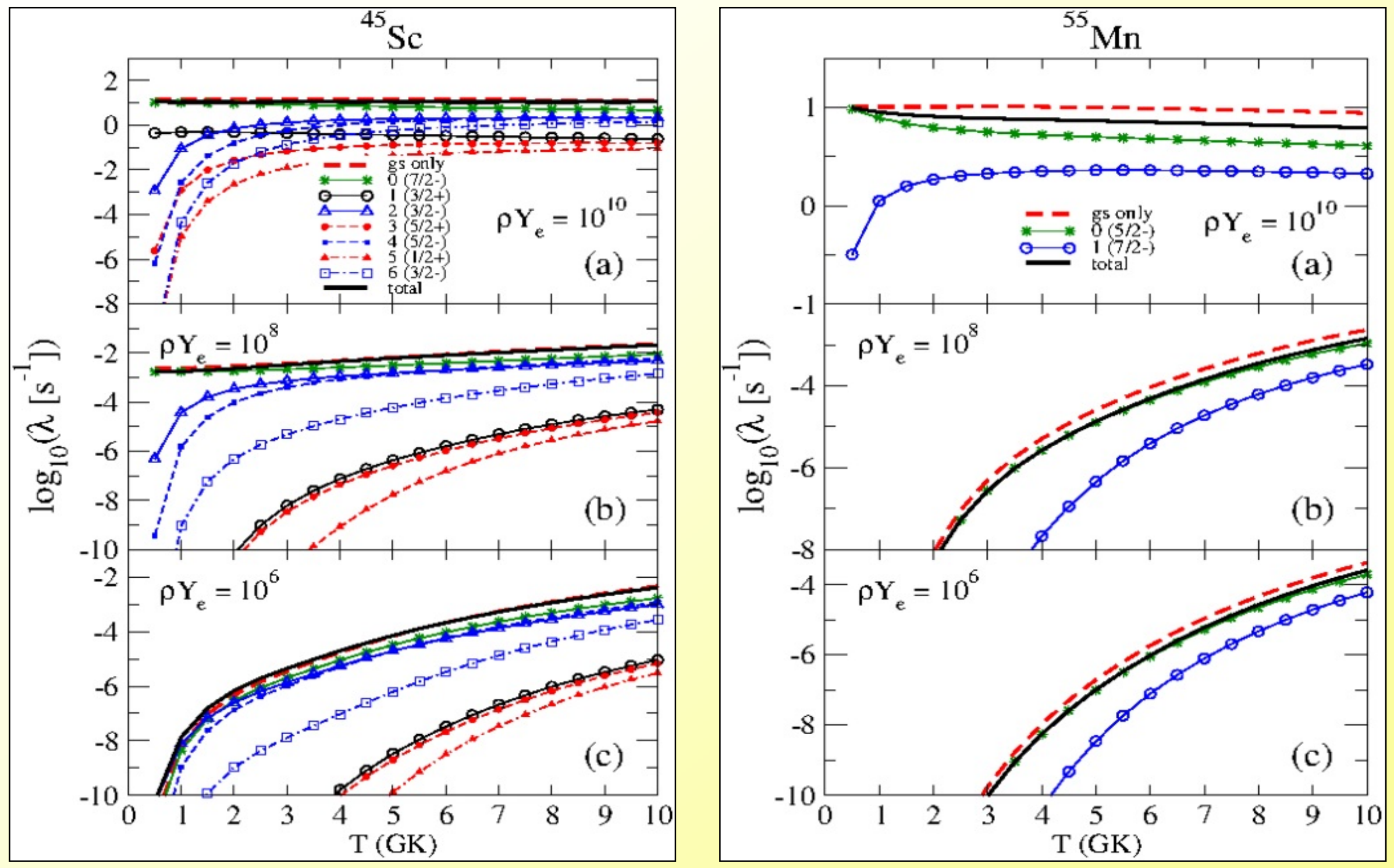